\title{
CONVERGENCE OF HARMONIC MAPS
}

\author{
ZAHRA SINAEI
}

\begin{abstract}
In this paper we prove a compactness theorem for sequences of harmonic maps which are defined on converging sequences of Riemannian manifolds.
\end{abstract}

Harmonic maps are critical points of the energy functional defined on the space of maps between Riemannian manifolds. This theory was developed by J. Eells and H. Sampson [ES64] in the 1960s. The notion of harmonic maps on smooth metric measure spaces was introduced by Lichnerowicz in Lic69]. Harmonic maps betweens singular spaces have been studied since the early 1990s in the works of Gromov-Schoen in [GS92] and KorevaarSchoen in [KS93. Eells and Fuglede describe the application of the methods of [KS93] to the study of maps between polyhedra EF01.

A smooth metric measure space is a triple $\left(M, g, \Phi \mathrm{dvol}_{M}\right)$, where $(M, g)$ is an $n$ dimensional Riemannian manifold, $\mathrm{dvol}_{M}$ denotes the corresponding Riemannian volume element on $M$, and $\Phi$ is a smooth positive function on $M$. These spaces have been used extensively in geometric analysis and they arise as smooth collapsed measured GromovHausdorff limits in the works of Cheeger-Colding [CC97, CC00a, CC00b], Fukaya [Fuk87] and Gromov Gro81. They have been studied recently by Morgan Mor05. See also works of Lott Lot03, Qian Qia97, Fang-Li-Zhang [FLZ09], Wei-Wylie [WW09, Wu Wu10], Su-Zhang [SZ11] and Munteanu-Wang [MW11].

In this paper, we are going to study the behavior of harmonic maps under convergence. Let $\mathcal{M}(n, D)$ denote the set of all compact Riemannian manifolds $(M, g)$ such that $\operatorname{dim}(M)=n, \operatorname{diam}(M)<D$, and the sectional curvature $\sec _{g}$ satisfies $\left|\sec _{g}\right| \leq 1$, equipped with the measured Gromov-Hausdorff topology. Let $\left(M_{i}, g_{i}, \mathrm{dvol}_{M_{i}}\right)$ in $\mathcal{M}(n, D)$ be a sequence of manifolds which converges to a smooth metric measure space $\left(M, g, \Phi \mathrm{dvol}_{M}\right)$. Suppose $f_{i}:\left(M_{i}, g_{i}\right) \rightarrow(N, h)$ is a sequence of harmonic maps. We are interested in knowing under what circumstances the $f_{i}$ converge to a harmonic map $f$ on the smooth metric measure space $\left(M, g, \Phi \mathrm{dvol}_{M}\right)$.

When a sequence of manifolds $\left(M_{i}, g_{i}\right)$ in $\mathcal{M}(n, D)$ converges to a metric space $X$, according to Fukaya [Fuk88], $X$ is a quotient space $Y / O(n)$, where $Y$ is a smooth manifold. Indeed $Y$ is the limit point of the sequence of frame bundles, $F\left(M_{i}\right)$, over the manifolds $M_{i}$ and $X$ has the structure of a Riemannian polyhedron $\left(X, g_{X}, \Phi_{X} \mu_{g}\right)$ where $\mu_{g}$ is the Riemannian volume element related to the metric $g_{X}$ on $X$.

We state the main result of this paper which is a compactness theorem for sequences of harmonic maps.

Theorem 0.1. . Let $\left(M_{i}, g_{i}\right)$ be a sequence of smooth Riemannian manifolds in $\mathcal{M}(n, D)$ which converges to a metric measure space $\left(X, g, \Phi \mu_{g}\right)$ in the measured Gromov-Hausdorff 
Topology. Suppose $(N, h)$ is a compact Riemannian manifold. Let $f_{i}:\left(M_{i}, g_{i}\right) \rightarrow(N, h)$ be a sequence of harmonic maps such that $\left\|e_{g_{i}}\left(f_{i}\right)\right\|_{L^{\infty}}<C$, where $\left\|e_{g_{i}}\left(f_{i}\right)\right\|_{L^{\infty}}$ is the $L^{\infty}$. norm of the energy density of the map $f_{i}$ and $C$ is a constant independent of $i$. Then $f_{i}$ has a subsequence which converges to a map $f:\left(X, g, \Phi \mu_{g}\right) \rightarrow(N, h)$, and this map is a harmonic map in $\mathcal{H}^{1}\left(\left(X, \Phi \mu_{g}\right), N\right)$.

By $\mathcal{H}^{1}(X, N)$ we mean

$$
\left\{f \in \mathcal{H}^{1}\left(X, \mathbb{R}^{q}\right) \mid f(x) \in N \text { for almost all } x \in M\right\},
$$

where $\mathcal{H}^{1}\left(X, \mathbb{R}^{q}\right)$ is the standard Sobolev space and $N$ is isometrically embedded in $\mathbb{R}^{q}$. In this work we use the notations $\mathcal{H}^{1}$ and $W^{1,2}$ interchangeably. For the notion of convergence of maps we refer the reader to the Definition 1.11.

The rest of this paper is organized as follows. In the first section we introduce our main notations and preliminary results needed for the rest of this paper. In the second section, we prove Theorem 0.1. We divide the proof into three cases. In Subsection 2.1 we consider the non-collapsing case, Proposition 2.1. Moreover using the regularity results for harmonic maps in the work of Schoen and Lin [Sch84, Lin99] we study Theorem 0.1 under less restrictive assumption of uniform boundedness of the energy of the maps $f_{i}$ (see Propositions 2.3, 2.4). In subsection 2.2 we consider the case of collapsing to a Riemannian manifold, Proposition 2.5. As a preliminary step we prove the result under some regularity assumption on the metrics $g_{i}$, see Proposition 2.6. The general case is considered in subsection 2.3. The Appendix is devoted to the study of convergence of the tension fields of the maps $f_{i}$ under the assumptions of Proposition 2.6.

\section{ACKNOWLEDGEMENT}

This work is part of my Ph.D. dissertation. I thank my advisor Professor Marc Troyanov for his guidance and support in the completion of this work. I also thank Professors Buser, Naber, and Wenger for their reading of this document and their comments and suggestions.

\section{BACKGROUND}

1.1. Harmonic maps. In this subsection, we first recall the definition of weakly harmonic maps on smooth metric measure spaces. We then briefly review this concept on Riemannian polyhedra. At the end we present some theorems and lemmas that we need in this paper. Let $(N, h)$ be a compact Riemannian manifold and $I$ an isometric embedding $I: N \rightarrow \mathbb{R}^{q}$. Since $I(N)$ is a smooth, compact submanifold of $\mathbb{R}^{q}$, there exists a number $\kappa>0$ such that the neighborhood

$$
U_{\kappa}(N)=\left\{y \in \mathbb{R}^{q}: \operatorname{dist}(y, N)<\kappa\right\}
$$

has the following property: for every $y$ in $U_{\kappa}(N)$ there exists a unique point $\pi_{N}(y) \in N$ such that

$$
\left|y-\pi_{N}(y)\right|=\operatorname{dist}(y, N)
$$

The map $\pi_{N}: U_{\kappa}(N) \rightarrow N$ defined as above is called the nearest point projection onto $N$. 
The Hess $\pi_{N}$ defines an element in $\Gamma\left(T N^{*} \otimes T N^{*} \otimes T N^{\perp}\right)$ which coincides with the second fundamental form of $I: N \rightarrow \mathbb{R}^{q}$ up to a negative sign

$$
\left\langle\operatorname{Hess} \pi_{N}(y)(X, Y), \eta\right\rangle=-\left\langle\nabla_{Y} \eta, X\right\rangle
$$

where $X$ and $Y$ are in $T N, y$ in $N$ and $\eta$ in $T N^{\perp}$ (see $\S 3$ in Moser [Mos05]).

A map $f:\left(M, g, \Phi \mathrm{dvol}_{M}\right) \rightarrow(N, h)$, belonging to $\mathcal{H}_{l o c}^{1}\left(\left(M, \Phi \operatorname{dvol}_{M}\right), N\right)$ is called weakly harmonic if and only if

$$
\Delta I \circ f-\Pi(f)(d f, d f)+d I \circ f(\nabla \ln (\Phi))=0
$$

in the weak sense. Here

$$
\Pi(f)(d f, d f)=\operatorname{trace} \operatorname{Hess}\left(\pi_{N}\right)(I \circ f)(d I \circ f, d I \circ f),
$$

or in coordinates

$$
\Pi(f)(d f, d f)=\sum g^{i j} \frac{\partial^{2} \pi_{N}^{A}}{\partial z^{B} \partial z^{C}} \frac{\partial f^{B}}{\partial x^{i}} \frac{\partial f^{C}}{\partial x^{j}} .
$$

For $f:\left(M^{n}, g\right) \rightarrow\left(N^{m}, h\right)$ and $\eta: M \rightarrow \mathbb{R}^{q}$, we define

$$
\Xi_{g}(f, \eta)=\langle d I \circ f, d \eta\rangle-\langle\Pi(f)(d f, d f), \eta\rangle .
$$

We explain now what we mean by harmonic maps on Riemannian polyhedra. Following Eells-Fuglede [EF01 on an admissible Riemannian polyhedron $X$, a continuous weakly harmonic map $u:\left(X, g, \mu_{g}\right) \rightarrow(N, h)$ is of class $\mathcal{H}_{l o c}^{1}(X, N)$ and satisfies: for any chart $\eta: V \rightarrow \mathbb{R}^{n}$ on $N$ and any open set $U \subset u^{-1}(V)$ of compact closure in $X$, the equality

$$
\int_{U} g\left(\nabla \lambda, \nabla u^{k}\right) d \mu_{g}=\int_{U} \lambda\left(\Gamma_{\alpha \beta}^{k} \circ u\right) g\left(\nabla u^{\alpha}, \nabla u^{\beta}\right) d \mu_{g}
$$

holds for every $k=1, \ldots, n$ and every bounded function $\lambda \in \mathcal{H}_{0}^{1}(U)$. Here $\Gamma_{\alpha \beta}^{k}$ denote the Christoffel symbols on $N$. Similarly on a polyhedron $X$ with a measure $\Phi \mu_{g}$, a continuous weakly harmonic map is a map in $\mathcal{H}_{l o c}^{1}\left(\left(X, \Phi \mu_{g}\right), N\right)$ which satisfies equation (4) with $\Phi d \mu_{g}$ in place of $d \mu_{g}$. When the target is compact a continuous map $f$ on an admissible Riemannian polyhedron is harmonic if and only if it satisfies (11) weakly.

Theorem 1.1 (Moser [Mos05], Theorem 3.1). Let $f \in \mathcal{H}^{1}(U, N) \cap C^{0}(U, N)$ be a weakly harmonic map, where $U$ is an open domain in $\mathbb{R}^{n}$. Then $f$ is smooth.

The energy functional is lower semi continuous, and we have

Lemma 1.2 (Xin Xin96]). Let $S \subset \mathcal{H}^{1}(M, N)$ be such that the energy functional is bounded on $S$ and $S$ is closed under weak limits. Then $S$ is sequentially compact.

Now we recall some regularity results for harmonic maps from [Sch84] and [Lin99]. Let $M$ and $N$ be compact Riemannian manifolds. Define

$$
\mathcal{F}_{\Lambda}=\left\{u \in C^{\infty}(M, N): u \text { is harmonic and } E(u) \leq \Lambda\right\} .
$$

We have the following results. 
Theorem 1.3 (Schoen [Sch84]). Let $M$ and $N$ be compact Riemannian manifolds. Any map $u$ in the weak closure of $\mathcal{F}_{\Lambda}$ is smooth and harmonic outside a relatively closed singular set of locally finite Hausdorff $(n-2)$-dimensional measure.

Remark 1 (Schoen [Sch84], Lin [Lin99]). Let $u_{i}$ be a sequence in $\mathcal{F}_{\Lambda}$. Then there exists a subsequence which converges weakly to some $u$ in $\mathcal{H}^{1}(M, N)$. Define

$$
\Sigma=\bigcap_{r>0}\left\{x \in M, \liminf _{i \rightarrow \infty} r^{2-n} \int_{B_{r}(x)} e\left(u_{i}\right) \geq \epsilon_{0}\right\}
$$

where $\epsilon_{0}=\epsilon_{0}(n, N)>0$ is a constant independent of $u_{i}$ as in Theorem 2.2 in [Sch84]. If we consider a sequence of Radon measures $\mu_{i}=\left|d u_{i}\right|^{2} d x$, without loss of generality we may assume $\mu_{i} \rightarrow \mu$ weakly as Radon measures. By Fatou's lemma, we may write

$$
\mu=|d u|^{2} d x+\nu
$$

for some non-negative Radon measure $\nu$. We can show that $\Sigma=\operatorname{spt} \nu \cup \operatorname{sing} u$ and $\nu$ is absolutely continuous with respect to $\left.H^{n-2}\right|_{\Sigma}$. Therefore $u_{i}$ converges strongly in $\mathcal{H}^{1}(M, N)$ to $u$ if and only if $\left|d u_{i}\right|^{2} d x \rightarrow|d u|^{2} d x$ weakly, if and only if $\nu=0$, if and only if $H^{n-2}(\Sigma)=0$, if and only if there is no smooth non-constant harmonic map from 2 -sphere $\mathbb{S}^{2}$ into $N$, e.g. negatively curved manifolds. See Lemma 3.1 in [Lin99] for a complete discussion.

The following reduction theorem shows the relation between the tension fields of equivariant harmonic maps under Riemannian submersions.

Theorem 1.4 (Xin Xin96, Theorem 6.4). Let $\pi_{1}: E_{1} \rightarrow M_{1}$ and $\pi_{2}: E_{2} \rightarrow M_{2}$ be Riemannian submersions, $H_{1}$ the mean curvature vector of the submanifold $F_{1}$ in $E_{1}$ and $B_{2}$ the second fundamental form of the fiber submanifold $F_{2}$ in $E_{2}$. Let $f: E_{1} \rightarrow E_{2}$ be a horizontal equivariant map and $\bar{f}$ its induced map from $M_{1}$ to $M_{2}$ with tension field $\tau(\bar{f})$. Let $f^{\perp}$ be the restriction of $f$ to the fiber $F_{1}$. Then we have the following formula

$$
\tau(f)=\tau^{*}(\bar{f})+B_{2}\left(f_{*}\left(e_{t}\right), f_{*}\left(e_{t}\right)\right)-f_{*}\left(H_{1}\right)+\tau\left(f^{\perp}\right)
$$

where $\left\{e_{t}\right\}, t=n_{1}+1, \ldots, m_{1}$ is a local orthonormal frame field on the fiber $F_{1}$ and $\tau^{*}(\bar{f})$ denotes the horizontal lift of $\tau(\bar{f})$.

1.2. Hölder spaces on manifolds. Let $(M, g)$ be a Riemannian manifold and let $\nabla$ be the Levi-Civita connection on $M$. Let $V$ be a vector bundle on $M$ equipped with the Euclidean metric on its fibers. Let $\hat{\nabla}$ be a connection on $V$ preserving these metrics. Let $C^{k}(M)$ be the space of all continuous, bounded functions $f$ that have $k$ continuous, bounded derivatives and define the norm $\|\cdot\|_{C^{k}}$ on $C^{k}(M)$ by $\|f\|_{C^{k}}=\sum_{j=0}^{k} \sup _{M}\left|\nabla^{j} f\right|$.

Now we define the Hölder space $C^{0, \alpha}(M)$ for $\alpha \in(0,1)$. The function $f$ on $M$ is said to be Hölder continuous with exponent $\alpha$, if

$$
[f]_{\alpha}=\sup _{x \neq y \in M} \frac{|f(x)-f(y)|}{d(x, y)^{\alpha}}
$$

is finite. The vector space $C^{0, \alpha}(M)$ is the set of continuous, bounded functions on $M$ which are Hölder continuous with exponent $\alpha$ and the norm $C^{0, \alpha}(M)$ is $\|f\|_{C^{0, \alpha}}=\|f\|_{C^{0}}+[f]_{\alpha}$. 
In the same way, we shall define Hölder norms on spaces of sections $v$ of a vector bundle $V$ over $M$ equipped with Euclidean metrics in the fibers as above. Let $\delta(g)=\operatorname{injrad}(M, g)$ be the injectivity radius of the metric $g$ on $M$ which we suppose to be positive and set

$$
[v]_{\alpha}=\sup _{\substack{x \neq y \in M \\ d(x, y)<\delta(g)}} \frac{|v(x)-v(y)|}{d(x, y)^{\alpha}}
$$

We now interpret $|v(x)-v(y)|$. When $x \neq y \in M$, and $d(x, y) \leq \delta(g)$, there is unique geodesic $\gamma$ of length $d(x, y)$ joining $x$ and $y$ in $M$. Parallel translation along $\gamma$ using $\hat{\nabla}$ identifies the fibers of $V$ over $x$ and $y$ and the metrics on the fibers. With this understanding the expression $|v(x)-v(y)|$ is well defined.

Define $C^{k, \alpha}(M)$ to be the set of $f$ in $C^{k}(M)$ for which $\left[\nabla^{k} f\right]_{\alpha}$ defined by (5) exists as a section in the vector bundle $\bigotimes^{k} T^{*} M$ with its natural metric and connection. The Hölder norm on $C^{k, \alpha}(M)$ is $\|f\|_{C^{k, \alpha}}=\|f\|_{C^{k}}+\left[\nabla^{k} f\right]_{\alpha}$.

Lemma 1.5. Let $\Omega \subset \mathbb{R}^{n}$ be a bounded domain. Suppose that $F: \Omega \rightarrow \mathbb{R}^{q}$ is bounded and Hölder continuous. Let $Q: \mathbb{R}^{q} \rightarrow \mathbb{R}^{p}$ be a quadratic function. Then $Q \circ F: \Omega \rightarrow \mathbb{R}^{p}$ is also Hölder continuous and

$$
[Q \circ F]_{\alpha} \leq A \sup _{\Omega}\|F\|_{\mathbb{R}^{q}}\left[\|F\|_{\mathbb{R}^{q}}\right]_{\alpha}
$$

where $A$ is a constant.

In the above lemma by a quadratic function we mean

$$
Q(y)=\sum_{i, j=1}^{q} Q_{i j} y_{i} y_{j}, \quad Q_{i j} \in C^{1}(\bar{\Omega}) .
$$

We have

Corollary 1.6. Let $f \in C^{1, \alpha}(M, N)$, then

$$
[\Pi(f)(d f, d f)]_{C^{\alpha}} \leq A \cdot\|d f\|_{L^{\infty}} \cdot[d f]_{C^{\alpha}} .
$$

Proof. Let $\left\{\Omega_{j}\right\}$ be an atlas of $M$, such that $\operatorname{diam}\left(\Omega_{j}\right) \leq \operatorname{injrad}(M)$ and set $F_{j}=\left.d f\right|_{\Omega_{j}}$ and $Q=\operatorname{Hess} \pi_{N}(X, X)$, for an smooth vector field $X$. Then using the previous lemma and an appropriate partition of unity we will have the result.

Schauder Estimates. In this part, we give a quick review on the Schauder estimate of solutions to linear elliptic partial differential equations. Suppose $(M, g)$ is compact and $L$ is an elliptic operator, $L=a^{i j} \nabla_{i} \nabla_{j}+b_{i} \nabla_{i}+c$, where $a$ is a symmetric and positive definite tensor, $b$ is a $C^{0, \alpha}$ vector field on $M$ and $c$ is in $C^{0, \alpha}(M)$ such that $L$ satisfies the conditions

$$
\begin{gathered}
\|a\|_{C^{0, \alpha}}+\|b\|_{C^{0, \alpha}}+\|c\|_{C^{0, \alpha}} \leq \Lambda, \\
\lambda\|\xi\|^{2} \leq a^{i j}(x) \xi_{i} \xi_{j} \leq \Lambda\|\xi\|^{2}, \quad \text { for all } x \in M, \text { and } \xi \in \mathbb{R}^{n} .
\end{gathered}
$$


Consider the following problem,

$$
L u=f \quad \text { in } M
$$

if $\partial M=\emptyset$ and

$$
\begin{cases}L u=f & \text { in } M \\ u=g & \text { on } \partial M\end{cases}
$$

if $\partial M \neq \emptyset$. Then we have (c.f. Gilbarg-Trudinger [GT83])

Theorem 1.7 (Schauder Estimate). If $f \in C^{0, \alpha}(M)$ and $u \in C^{2}(M)$, then $u \in C^{2, \alpha}(M)$ and we have

$$
\begin{aligned}
\|u\|_{C^{1, \alpha}} & \leq C\left(\|f\|_{L^{\infty}}+\|u\|_{L^{\infty}}\right), \\
\|u\|_{C^{2, \alpha}} & \leq C\left(\|f\|_{C^{0, \alpha}}+\|u\|_{L^{\infty}}\right),
\end{aligned}
$$

where $C$ depends on $M, \lambda, \Lambda$.

Hereafter we present an introduction to the convergence and collapsing theory. Most of the materials in this part was gathered from the work of Rong [Ron10].

1.3. Convergence. Gromov introduced the notion of the Gromov-Hausdorff distance between metric spaces in Gro81]), based on the notion of Hausdorff distance between subsets $A, B$ in a metric space $Z$ :

$$
d_{H}^{Z}(A, B)=\inf \left\{\epsilon>0: B \subset T_{\epsilon}(A) \text { and } A \subset T_{\epsilon}(B)\right\}
$$

where $T_{\epsilon}(A)=\left\{x \in Z: d_{Z}(x, A)<\epsilon\right\}$ is a tubular neighborhood of a set $A$.

Definition 1.8. (Gromov [Gro81]) Let $X$ and $Y$ be two compact metric spaces. The Gromov-Hausdorff distance between $X$ and $Y$ is defined as

$d_{G H}(X, Y)=\inf \left\{d_{H}^{Z}(\phi(X), \psi(Y)): \begin{array}{c}\text { for all metric spaces } Z \text { and isometric embeddings } \\ \phi: X \hookrightarrow Z, \psi: Y \hookrightarrow Z\end{array}\right\}$

Let $\mathcal{M E T}$ denote the set of all isometry classes of nonempty compact metric spaces. Then $\left(\mathcal{M E} \mathcal{T}, d_{G H}\right)$ is a complete metric space. There is an alternative definition for Gromov-Hausdorff distance given in Gro81:

Definition 1.9. (Gromov [Gro81]) Let $X$ and $Y$ be two elements of $\mathcal{M E} \mathcal{T}$. A map $\phi$ : $X \rightarrow Y$ is said to be an $\epsilon$-Hausdorff approximation from $X$ to $Y$, if the following two conditions are satisfied

i. $\epsilon$-onto: $B_{\epsilon}(\phi(X))=Y$.

ii. $\epsilon$-isometry: $|d(\phi(x), \phi(y))-d(x, y)|<\epsilon$ for all $x, y \in X$.

The Gromov-Hausdorff distance $\hat{d}_{G H}(X, Y)$, between $X$ and $Y$ is defined to be the infimum of the positive number $\epsilon$ such that there exists $\epsilon$-Hausdorff approximation from $X$ to $Y$ and form $Y$ to $X$. 
The distance $\hat{d}_{G H}$ does not satisfy triangle inequality and $\hat{d}_{G H} \neq d_{G H}$ but onecan show that

$$
\frac{2}{3} d_{G H} \leq \hat{d}_{G H} \leq 2 d_{G H}
$$

Because a sequence in $\mathcal{M E} \mathcal{T}$ converges with respect to $d_{G H}$ if and only if it converges with respect to $\hat{d}_{G H}$, we will not distinguish $\hat{d}_{G H}$ from $d_{G H}$.

For the notion of equivariant Gromov-Hausdorff convergence and equivariant measured Gromov-Hausdorff convergence, we refer the reader to Definition 1.5.2 in Ron10 and Definition 3.11 in [Fuk87. Also for the notion of Lipschitz distance see Definition 3.1 in Gro81. Let $\mathcal{M M}$ denotes the class of all pairs $(X, \mu)$ of compact metric spaces $X$ equipped with a Borel measure $\mu$ on it such that $\mu(X)=1$. Fukaya in [Fuk87] presented a notion of measured Gromov-Hausdorff convergence for the metric measure spaces:

Definition 1.10. (Fukaya Fuk87]) Let $\left(X_{i}, \mu_{i}\right)$ be a sequence in $\mathcal{M M}$. We say that $\left(X_{i}, \mu_{i}\right)$ converges to an element $(X, \mu)$ in $\mathcal{M M}$ with respect to measured Gromov-Hausdorff topology if there exist Borel measurable $\epsilon$-Hausdorff approximations $f_{i}:\left(X_{i}, \mu_{i}\right) \rightarrow(X, \mu)$ such that $f_{i *}\left(\mu_{i}\right)$ converges to $\mu$ in the weak* topology.

When $M$ is a Riemannian manifold with finite volume, we let $\mu_{M}=\frac{\operatorname{dvol}_{M}}{\operatorname{vol}(M)}$, where $\operatorname{dvol}_{M}$ denotes the volume element of $M$ and regard $\left(M, \mu_{M}\right)$ as an element in $\mathcal{M M}$.

In GP91, Grove and Petersen introduced the notion of convergence of maps.

Definition 1.11. (Grove-Petersen GP91] Let $\left(X_{i}, p_{i}\right),(X, p),\left(Y_{i}, q_{i}\right)$ and $(Y, q)$ be pointed metric spaces such that $\left(X_{i}, p_{i}\right)$ converges to $(X, p)$ in the pointed Gromov-Hausdorff topology (resp. $\left(Y_{i}, q_{i}\right)$ converges to $\left.(Y, q)\right)$. We say that a sequence of maps $f_{i}:\left(X_{i}, p_{i}\right) \rightarrow$ $\left(Y_{i}, q_{i}\right)$ converges to a map $f:(X, p) \rightarrow(Y, q)$ if there exists a subsequence $X_{i_{k}}$ such that if $x_{i_{k}} \in X_{i_{k}}$ and $x_{i_{k}}$ converges to $x$ (in $\amalg X_{i_{k}} \amalg X$ with the admissible metric), then $f_{i_{k}}\left(x_{i_{k}}\right.$ ) converges to $f(x)$.

A family of maps $f_{i}:\left(X_{i}, d_{X_{i}}, p_{i}\right) \rightarrow\left(Y_{i}, d_{Y_{i}}, q_{i}\right)$ is called equicontinuous if for any $\epsilon>0$ there is $\delta>0$ such that $d_{X_{i}}\left(x_{i}, y_{i}\right)<\delta$ implies $d_{Y_{i}}\left(f_{i}\left(x_{i}\right), f_{i}\left(y_{i}\right)\right)<\epsilon$ for all $x_{i}, y_{i}$ in $X_{i}$ and for all $i$. We have

Lemma 1.12. (Grove-Petersen GP91]) Let $\left(X_{i}, p_{i}\right),(X, p),\left(Y_{i}, q_{i}\right)$ and $(Y, q)$ be pointed metric spaces such that $\left(X_{i}, p_{i}\right)$ converges to $(X, p)$ in the pointed Gromov-Hausdorff topology (resp. $\left(Y_{i}, q_{i}\right)$ converges to $\left.(Y, q)\right)$. Let $f_{i}:\left(X_{i}, p_{i}\right) \rightarrow\left(Y_{i}, q_{i}\right)$ be a sequence of maps. Then

i. If $f_{i}$ s are equicontinuous, then there is a uniformly continuous map $f$ and a convergent subsequence $X_{i_{k}}$ such that $f_{i}$ converges to $f$.

ii. If $f_{i}$ s are isometries then the limit map $f:(X, p) \rightarrow(Y, q)$ is also an isometry.

1.4. Convergence Theorems, Non-Collapsing. This subsection is devoted to the theory of convergence of manifolds in the non-collapsing case. A sequence of $n$-manifolds $M_{i}$ converging to a metric space $X$ is called non-collapsing if $\operatorname{vol}\left(M_{i}\right) \geq v>0$, and collapsing otherwise. For a non-collapsing sequence of manifolds with bounded sectional curvature 
there is a uniform lower bound on the injectivity radius of $M_{i}$, and thus $M_{i}$ s are diffeomorphic for large $i$. This result is due to Cheeger-Gromov (Cheeger Che70, Peters [Pet84, Greene-Wu [GW88]) and is formulated as follows.

Theorem 1.13. Let $\left(M_{i}, g_{i}\right)$ be a sequence of closed Riemannian n-manifolds such that $\left|\sec _{g_{i}}\right| \leq 1$ and $\operatorname{vol}\left(M_{i}\right) \geq v>0$, and $M_{i}$ converges to a metric space $X$. Then $X$ is homeomorphic to a manifold $M$ such that for large $i$, and there are diffeomorphisms $\phi_{i}: M \rightarrow M_{i}$ such that the pullback metric converges to a $C^{1, \alpha}$-metric $g$ on $M$ in the $C^{1, \alpha}$-topology.

The following smoothing result concerns the uniform approximation of Riemannian manifolds by smooth ones.

Theorem 1.14 (Bemelmans-Oo-Ruh [BMOR84]). Let $(M, g)$ be a compact Riemannian $n$-manifold with $\left|\sec _{g}\right|<1$. For any $\epsilon>0$, there is a smooth metric $g_{\epsilon}$ on $M$ such that

$$
\left|g_{\epsilon}-g\right|_{C^{1}}<\epsilon, \quad\left|\sec _{g_{\epsilon}}\right| \leq 1, \quad\left|\nabla^{k} \mathrm{R}_{g_{\epsilon}}\right| \leq C(n, k) \cdot \epsilon^{k} .
$$

In particular

$$
\begin{gathered}
\mathrm{e}^{-\epsilon} \operatorname{injrad}(M, g) \leq \operatorname{injrad}\left(M, g_{\epsilon}\right) \leq \mathrm{e}^{\epsilon} \operatorname{injrad}(M, g), \\
\mathrm{e}^{-\epsilon} \operatorname{diam}(M, g) \leq \operatorname{diam}\left(M, g_{\epsilon}\right) \leq \mathrm{e}^{\epsilon} \operatorname{diam}(M, g), \\
\mathrm{e}^{-\epsilon} \operatorname{vol}(M, g) \leq \operatorname{vol}\left(M, g_{\epsilon}\right) \leq \mathrm{e}^{\epsilon} \operatorname{vol}(M, g) .
\end{gathered}
$$

1.5. Convergence Theorems-Collapsing. This subsection is devoted to the theory of convergence of manifolds in the collapsing case. We state some of the main results in this context.

Theorem 1.15 (Fibration theorem, Fukaya [Fuk89, Cheeger-Fukaya-Gromov [CFG92]). Let $M^{n}$ and $N^{m}$ be compact Riemannian manifolds satisfying

$$
\sec _{M^{n}} \geq-1, \quad\left|\sec _{N^{m}}\right| \leq 1(m \geq 2), \quad \operatorname{injrad}\left(N^{m}\right) \geq i_{0}>0 .
$$

Assume $M^{n}$ and $N^{m}$ admit isometric compact Lie group $G$-actions. There exists a constant $\epsilon\left(n, i_{0}\right)>0$ such that if $d_{e q G H}\left(\left(M^{n}, G\right),\left(N^{m}, G\right)\right)<\epsilon \leq \epsilon\left(n, i_{0}\right)$, then there is a $C^{1}$-fibration $G$-invariant map, $f:\left(M^{n}, G\right) \rightarrow\left(N^{m}, G\right)$ with connected fibers such that

$i$. The diameter of any $f$-fibers is at most $c_{1} \cdot \epsilon$, where $c_{1}=c_{1}(n, \epsilon)$ is such that $c_{1} \rightarrow 1$ as $\epsilon \rightarrow 0$.

ii. $f$ is an almost Riemannian submersion, that is for any vector $\xi \in T M$ orthogonal to a fiber,

$$
\mathrm{e}^{-\tau(\epsilon)} \leq \frac{|d f(\xi)|}{|\xi|} \leq \mathrm{e}^{\tau(\epsilon)}
$$

where $\tau(\epsilon) \rightarrow 0$ as $\epsilon \rightarrow 0$.

iii. If in addition, $\sec _{M^{n}} \leq 1$ then $f$ is smooth and the second fundamental form of any fiber satisfies $\left|I I_{f^{-1}(\bar{x})}\right| \leq c_{2}(n)$, for $\bar{x}$ in $N^{m}$.

iv. The fibers are diffeomorphic to an infranilmanifold $\Gamma \backslash \stackrel{\circ}{N}$, where $\stackrel{\circ}{N}$ is a simply connected nilpotent group, $\Gamma \subset \stackrel{\circ}{N} \ltimes \operatorname{Aut}(\stackrel{N}{N})$, such that $[\Gamma, \stackrel{N}{N} \cap \Gamma] \leq \omega(n)$. 
An easily accessible proof of this theorem can be founded in Ron10 Theorems 2.1.1 and 5.7.1.

A pure nilpotent Killing structure on $M^{n}$ is a $G$-equivarient fibration $N_{0} \rightarrow M^{n} \rightarrow N^{m}$, with fiber $N_{0}$ a nilpotent manifold (equipped with a flat connection) on which parallel fields are Killing fields and the $G$-action preserves affine fibrations. The underlying $G$ invariant affine bundle structure is called a pure $N_{0}$-structure and a metric for which the $N_{0}$-structure becomes a nilpotent Killing structure is called invariant.

Let $M^{n}$ and $N^{m}$ be as in Theorem 1.15. Suppose $M^{n}$ and $N^{m}$ satisfy the following: for some sequence $A=\left\{A_{k}\right\}$ of real non-negative numbers, for the Riemannian curvature tensor on $M$ and $N$ we have

$$
\left|\nabla^{k} \mathrm{R}\right| \leq A_{k}
$$

We can construct an invariant metric (invariant under the left action of $N_{0}$ ) such that

$$
\left|\nabla^{k}(\langle, \quad\rangle-(\quad, \quad))\right| \leq c(n, A) \cdot \epsilon \cdot \operatorname{inj} \operatorname{rad}(N)^{-(k+1)},
$$

where $\langle, \quad\rangle$ denotes the original metric, $(, \quad)$ the invariant one, and $c(n, A)$ is a generic constant depending on finitely many $A_{k}$ and $n$. For the construction of invariant metric which satisfies inequality (7) see Proposition 4.9 in CFG92 and the explanation therein. Given such a metric we have a pure nilpotent killing structure.

When a sequence of Riemannian $n$-manifolds with bounded curvature collapses, the limit space can be a singular space. We have

Theorem 1.16 (Singular fibration theorem, Fukaya [Fuk88]). Let $\left(M_{i}, g_{i}\right)$ be a sequence of closed Riemannian n-manifolds with $\left|\sec _{g_{i}}\right| \leq 1$ and $\operatorname{diam}\left(M_{i}\right) \leq D$ which converges to the closed metric space $(X, d)$ in $\mathcal{M E} \mathcal{T}$. Then

$i$. The frame bundles equipped with canonical metrics converge, $\left(F\left(M_{i}\right), O(n)\right) \rightarrow(Y, O(n))$, where $Y$ is a manifold.

ii. There is an $O(n)$-invariant fibration $\tilde{f}_{i}: F\left(M_{i}\right) \rightarrow Y$ satisfying the conditions in Theorem 1.15 which becomes for $\epsilon>0$, a nilpotent Killing structure with respect to an $\epsilon C^{1}$-closed metric (with respect to $C^{1}$-topology). Moreover each fiber on $M_{i}$ has positive dimension.

iii. For any $\bar{x} \in X$, a fiber $f_{i}^{-1}(\bar{x})$ is singular if and only if $p^{-1}(\bar{x})$ is a singular $O(n)$-orbit in $Y$.

For the proof see Theorem 4.1.3 in [Ron10]. In the above theorem, the fibration map $\tilde{f}_{i}$ descends to a (singular) fibration map $f_{i}: M_{i} \rightarrow X=Y / O(n)$ such that the following diagram commutes

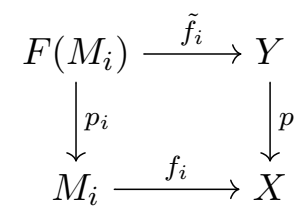


In the following remark we collect the main points that we need from the above theorems and explain the classification in the proof of Theorem 0.1 .

Remark 2. When a sequence of Riemannian manifolds $M_{i}$ converges in $\mathcal{M}(n, D)$ to a metric space $X$, the frame bundles over $M_{i}$ equipped with the canonical metrics $\tilde{g}_{i}$ converge to a manifold $Y$ and $\tilde{f}_{i}:\left(F\left(M_{i}\right), \tilde{g}_{i}, O(n)\right) \rightarrow(Y, O(n))$ is an $O(n)$-invariant fibration map.

To see this let $\tilde{g}_{i \epsilon}$ be the smooth metric on $F\left(M_{i}\right)$ as in Theorem 1.14, Then $\left(F\left(M_{i}\right), \tilde{g}_{i_{\epsilon}}\right)$ converges to a smooth Riemannian manifold $\left(Y_{\epsilon}, g_{\epsilon}\right)$. For a small fixed $\epsilon_{0}$ and $\epsilon<\epsilon_{0}$, the sectional curvature on $\left(F\left(M_{i}\right), \tilde{g}_{i_{\epsilon}}\right)$ is uniformly bounded and we can apply Theorem 1.15 to conclude that there exists an $O(n)$-invariant smooth fibration map $\tilde{f}_{i_{\epsilon}}$. By continuity $\left(F\left(M_{i}\right), \tilde{g}_{i_{\epsilon}}\right)$ is conjugate to $\left(F\left(M_{i}\right), \tilde{g}_{i_{0}}\right)$ (by being conjugate we mean there exists $C^{1, \alpha_{-}}$ diffeomorphism as in Theorem 1.13). This implies that the convergence of $Y_{\epsilon}$ to $Y$ is the same as the convergence of a sequence of metrics on $Y_{\epsilon_{0}}$, and therefor $(Y, O(n))$ is conjugate to $\left(Y_{\epsilon_{0}}, O(n)\right)$

$$
\left(F\left(M_{i}\right), O(n)\right) \simeq\left(F\left(M_{i}\right), \tilde{g}_{i_{0}}, O(n)\right) \stackrel{\tilde{f}_{i_{\epsilon_{0}}}}{\rightarrow}\left(Y_{\epsilon_{0}}, O(n)\right) \simeq(Y, O(n)),
$$

and it induces a fibration map $\left(F\left(M_{i}\right), \tilde{g}_{i}, O(n)\right) \stackrel{\tilde{f}_{i}}{\rightarrow}(Y, O(n))$. For more explanations see the proof of Theorem 4.1.3 in Ron10.

Furthermore, there exists a $C^{1}$-close invariant Riemannian metric ${\stackrel{\circ}{g_{\epsilon}}}_{i_{\epsilon}}$ such that $\left(F\left(M_{i}\right), \stackrel{\circ}{i_{i}}, O(n)\right)$ is a pure nilpotent Killing structure and the fibration map $\tilde{f}_{i_{\epsilon}}$ is a Riemannian submersion considering the induced Riemannian metric on $Y_{\epsilon}$ by this map.

1.6. Density function. Let $\mathcal{D} \mathcal{M}(n, D)$ denote the closure of $\mathcal{M}(n, D)$ in $\mathcal{M M}$ with respect to the measured Gromov-Hausdorff topology. Then $\mathcal{D} \mathcal{M}(n, D)$ is compact with respect to the measured Gromov-Hausdorff topology. Let $\left(M_{i}, g_{i}, \frac{\operatorname{dvol}_{M_{i}}}{\operatorname{vol}\left(M_{i}\right)}\right) \in \mathcal{M}(n, D)$ be a sequence of manifolds which converges to a manifold $(M, g, \mu)$. Suppose $\psi_{i}: M_{i} \rightarrow M$ is the fibration map as in Theorem 1.13, For $x \in M$ we define

$$
\Phi_{i}=\frac{\operatorname{vol}\left(\psi_{i}^{-1}(x)\right)}{\operatorname{vol}\left(M_{i}\right)}
$$

then there exists $\Phi$ such that $\Phi=\lim _{i \rightarrow \infty} \Phi_{i}$ and $\mu$ is absolutely continuous with respect to $\mathrm{dvol}_{M}, \mu=\Phi \mathrm{dvol}_{M}$ (see $\S 3$ in [Fuk87]). For the general case when $(X, \mu) \in \mathcal{D} \mathcal{M}(n, D)$, we first recall a remark on quotient spaces. Below $S(B)$ denotes the singular part of $B$.

Remark 3 (Besse [Bes08]). Let $(M, g)$ be a Riemannian manifold and $G$ a closed subgroup of isometries of $M$. Assume that the projection $p: M \rightarrow M / G$ is a smooth submersion. Then there exists a unique Riemannian metric $\check{g}$ on $B=M / G$ such that $p$ is a Riemannian submersion (see Subsection 9.12 in [Bes08]).

We recall that using the general theory of slices for the action of a group of isometries on a Riemannian manifold, one can show that there always exists an open dense submanifold $U$ of $M$ (the union of the principle orbits), such that the restriction $\left.p\right|_{U}: U \rightarrow U / G$ is a smooth submersion.

Considering now $M / G$ as a Riemannian polyhedron and $\mu_{g}$ as its Riemannian volume element, the restriction of $\mu_{g}$ on $U / G$ is equal to $\operatorname{dvol}_{U / G}=\operatorname{dvol}_{B-S(B)}$. 
Now suppose $M_{i}$ in $\mathcal{M}(n, D)$ converges to a metric space $X$. We may assume that $F M_{i}$ with the induced $O(n)$-invariant metric $\tilde{g}_{i}$ converges to $\left(Y, g, \Phi_{Y} \cdot \mathrm{dvol}_{Y}\right)$ with respect to the $O(n)$-measured Gromov-Hausdorff topology and $g, \Phi_{Y}$ are $C^{1, \alpha}$-regular. Moreover, since $p_{i}: F\left(M_{i}\right) \rightarrow M_{i}$ is a Riemannian submersion with totally geodesic fibers, and since the fibers are isometric to each other, it follows that $\left(F M_{i}, \operatorname{dvol}_{F M_{i}}\right) / O(n)=\left(M_{i}, \operatorname{dvol}_{M_{i}}\right)$. Hence by equivariant Gromov-Hausdorff convergence $M_{i}$ converges to $(X, \nu)=\left(Y, \Phi_{Y} \mathrm{dvol}_{Y}\right) /$ $O(n)$ (see Theorem 0.6 in [Fuk87]), and by Remark 3

$$
\nu(S(X))=0
$$

For all $x$ in $X$ we let

$$
\Phi_{X}(x)=\int_{y \in p^{-1}(x)} \Phi_{Y}(y) \operatorname{dvol}_{p^{-1}(x)},
$$

where $p: Y \rightarrow X$ is the natural projection. For each open set $U$

$$
\nu(U)=\int_{U} \Phi_{X}(x) \operatorname{dvol}_{X-S(X)} .
$$

\section{Proof of the Convergence Theorem}

In this section we are going to prove Theorem 0.1 . In the following $\mathcal{M}(n, D)$ denotes the set of all compact Riemannian manifolds $(M, g)$ such that $\operatorname{dim}(M)=n, \operatorname{diam}(M)<D$ and the sectional curvature satisfies $\left|\sec _{g}\right| \leq 1$, and $\mathcal{M}(n, D, v)$ the set of Riemannian manifolds in $\mathcal{M}(n, D)$ with volume $\geq v$.

We split the proof in three cases:

Case I: Non-collapsing. $\left(M_{i}, g_{i}\right)$ converge to $(M, g)$ in $\mathcal{M}(n, D, v)$. We first consider the situation where $M_{i}=M$ and $g_{i}$ converges to a metric $g$ in $\mathcal{M}(n, D, v)$. Then we study the problem in the general case using Theorem 1.13 .

Case II: Collapsing to a manifold. $\left(M_{i}, g_{i}\right)$ converge to $(M, g)$ in $\mathcal{M}(n, D)$ with $g$ a $C^{1, \alpha}$-metric. We first consider the situation when $\left(M_{i}, g_{i}\right)$ satisfies an additional regularity assumption (see Assumption 1 below). Then we discuss the general case using the fact that there is always a sequence of metrics $g_{i}(\epsilon)$ on $M_{i}, C^{1}$-close to the the metric $g_{i}$ which satisfies Assumption 1 as explained in Remark 2 .

Case III: Collapsing to a singular space. $\left(M_{i}, g_{i}\right)$ converge to a metric space $(X, d)$ in $\mathcal{M}(n, D)$. When a sequence of manifolds $\left(M_{i}, g_{i}\right)$ converges in $\mathcal{M}(n, D)$ to a metric space $X$, the frame bundles over $M_{i}$ converge to a Riemannian manifold $Y$, with a $C^{1, \alpha}$-metric and we have $X=Y / O(n)$. The harmonic maps over $M_{i}$, induce harmonic maps over $F\left(M_{i}\right)$ and this case reduces to the study of harmonic maps on quotient spaces.

Hereafter we fix an isometric embedding $I: N \rightarrow R^{q}$ and we often denote the composition $I \circ f$ simply by $f$, unless we need to explicitly distinguish these two maps. 
2.1. Case I: Non-collapsing. In this subsection we prove

Proposition 2.1. Let $\left(M_{i}, g_{i}\right)$ be a sequence of Riemannian manifolds in $\mathcal{M}(n, D, v)$ which converges to a Riemannian manifold $(M, g)$ in the Gromov-Hausdorff topology. Suppose $(N, h)$ is a compact Riemannian manifold. Let $f_{i}:\left(M_{i}, g_{i}\right) \rightarrow(N, h)$ be a sequence of smooth harmonic maps such that $\left\|e_{g_{i}}\left(f_{i}\right)\right\|_{L^{\infty}}<C$, where $C$ is a constant independent of $i$. Then $f_{i}$ has a subsequence which converges to a map $f:(M, g) \rightarrow(N, h)$ and this map is a smooth harmonic map.

To go through the proof in this case, we first consider the situation when a sequence of metrics $g_{i}$ on a manifold $M$ converges to a Riemannian metric $g$.

Lemma 2.2. Let $g_{i}$ be a sequence of Riemannian metrics on a smooth manifold $M$ and suppose $\left(M, g_{i}\right)$ converge to $(M, g)$ in $\mathcal{M}(n, D, v)$. Suppose $f_{i}:\left(M, g_{i}\right) \rightarrow N$ is a sequence of smooth harmonic maps such that

$$
\left\|e_{g_{i}}\left(f_{i}\right)\right\|_{L^{\infty}}<C
$$

where $C$ is a constant independent of $i$. Then there exists a subsequence of $f_{i}$ which converges to some $f$ in the $C^{k}$-topology for any $k \geq 0$ and $f$ is also harmonic.

Proof. By Theorem 1.13, the metric $g_{i}$ converges to $g$ in $\mathcal{M}(n, D, v)$ in the $C^{1, \alpha}$-topology. Using Schauder estimates, $f_{i}$ s have bounded norm in $C^{k}(M)$ for every $k \geq 0$ and hence converge to a map $f \in C^{k}(M)$. We have

$$
\lim _{i \rightarrow \infty} \Delta_{g_{i}} f_{i}=\Delta_{g} f
$$

and

$$
\lim _{i \rightarrow \infty} \Pi\left(f_{i}\right)\left(d f_{i}, d f_{i}\right)=\Pi(f)(d f, d f)
$$

The above limits lead to harmonicity of $f$.

Using the above lemma we can prove Proposition 2.1.

Proof of Proposition 2.1. Since $M_{i}$ converges to $M$ in $\mathcal{M}(n, D, v)$, by Theorem 1.13 there is a diffeomorphism $\phi_{i}: M_{i} \rightarrow M$, such that the pushforward $\bar{g}_{i}=\phi_{i_{*}}\left(g_{i}\right)$ of the metrics $g_{i}$ on $M_{i}$ converges to a $C^{1, \beta}$-metric $g$. Since the map $\phi_{i}:\left(M_{i}, g_{i}\right) \rightarrow\left(M, \bar{g}_{i}\right)$ is an isometry

$$
e_{g_{i}}\left(f_{i}\right)=e_{\bar{g}_{i}}\left(\bar{f}_{i}\right)
$$

where $\bar{f}_{i}$ is the map $f_{i} \circ \phi_{i}^{-1} \cdot f_{i}$ is harmonic and so $\bar{f}_{i}$. Therefore all the assumptions of Lemma 2.2 are satisfied here and the proof of Theorem 0.1 in this case is complete.

In Lemma 2.2 if we replace the assumption of uniform boundedness of the energy density $\left\|e_{g_{i}}\left(f_{i}\right)\right\|_{L^{\infty}}<C$ with the assumption uniform bound on the energy $E_{g_{i}}\left(f_{i}\right)<C$, then the limiting map is not necessarily harmonic (see Theorem 1.3 and Remark 11). 
Proposition 2.3. Let $\left(M_{i}, g_{i}\right)$ be a sequence of manifolds in $\mathcal{M}(n, D, v)$ which converges to a Riemannian manifold $(M, g)$ in the measured Gromov-Hausdorff topology. Suppose $(N, h)$ is a compact Riemannian manifold which does not carry any harmonic 2-sphere $S^{2}$. Let $f_{i}:\left(M_{i}, g_{i}\right) \rightarrow(N, h)$ be a sequence of harmonic maps such that $E_{g_{i}}\left(f_{i}\right)<C$ where $C$ is a constant independent of $i$. Then $f_{i}$ has a subsequence which converges to a map $f:(M, g) \rightarrow(N, h)$, and this map is a weakly harmonic map.

Proof. With the same argument as in the proof of Proposition 2.1 we consider $f_{i}$ and $g_{i}$ to be on the manifold $M$. When we have a sequence of Riemannian manifolds $\left(M, g_{i}\right)$ which converges in $\mathcal{M}(n, d, v)$, the injectivity radius is bounded from below and $\mathrm{dvol}_{g_{i}}$ converges to $\mathrm{dvol}_{g}$ weakly. Therefore if $E_{g_{i}}\left(f_{i}\right)<C, C$ independent of $i$, then $E_{g}\left(f_{i}\right)$ is uniformly bounded. Adapting the proof of Remark 1 for our case, $f_{i}$ converges strongly in $\mathcal{H}^{1}$ to a map $f$. Also $\operatorname{Hess}\left(\pi_{N}\right)$ restricted to a neighborhood of $N$ is $\operatorname{Lipschitz}$ and $\operatorname{Hess}\left(\pi_{N}\right) \circ f_{i}$ converges to Hess $\left(\pi_{N}\right) \circ f$ in $\mathcal{H}^{1}$-norm (see Lemma 6.4 in Taylor's book [Tay00]) and so therefore $\Pi\left(f_{i}\right)\left(d f_{i}, d f_{i}\right)$ converges weakly to $\Pi(f)(d f, d f)$. We have the same for $\Delta f_{i}$ and so $f$ is a weakly harmonic map.

Under the assumptions of the above theorem one can show more and prove $f$ is stationary harmonic. Under stronger assumptions on $N$ or on the image of $f$, we can show that the limit map $f$ is strongly harmonic. These results are direct consequences of some of the theorems in Sch84.

Proposition 2.4. Let $\left(M_{i}, g_{i}\right)$ and $f_{i}$ be as in Proposition 2.3. Then the map $f$ is smooth harmonic, provided that $N$ is a compact Riemannian manifold and we have one of the following conditions:

$i .(N, h)$ is a non-positively curved Riemannian manifold.

ii. There is no strictly convex bounded function on $f(M)$.

Proof. i. See Proposition 2.1 in [Sch84].

ii. See Corollary 2.4 in $\underline{S}$ ch84.

2.2. Case II: Collapsing to a manifold. In this subsection we prove

Proposition 2.5. Let $\left(M_{i}, g_{i}\right)$ be a sequence of Riemannian manifolds in $\mathcal{M}(n, D)$ which converges to a Riemannian manifold $\left(M, g, \Phi \mathrm{dvol}_{M}\right)$ in the measured Gromov-Hausdorff topology with $C^{1, \alpha}$-pair $(g, \Phi)$. Suppose $(N, h)$ is a compact Riemannian manifold. Let $f_{i}:\left(M_{i}, g_{i}\right) \rightarrow(N, h)$ be a sequence of smooth harmonic maps such that $\left\|e_{g_{i}}\left(f_{i}\right)\right\|_{L^{\infty}}<C$, where $C$ is a constant independent of $i$. Then $f_{i}$ has a subsequence which converges to a map $f:\left(M, g, \Phi \mathrm{dvol}_{g}\right) \rightarrow(N, h)$, and this map is a weakly harmonic map.

Before we prove the proposition in general, we will prove the following proposition which has an additional regularity assumption. Then at the end of this subsection, we will apply this proposition to prove case II. Consider the following assumption, 
Assumption 1. Let the Riemannian metric $g_{i}$ be regular on $M_{i}$, i.e. there exists a sequence $C=\left\{C_{k}\right\}$ of positive number $C_{k}$ independent of $i$, such that

$$
\left|\nabla_{g_{i}}^{k} \mathrm{R}_{g_{i}}\right|<C_{k} .
$$

Suppose also that the Riemannian metric $g_{i}$ is an invariant metric with respect to the nil-structure.

We have

Proposition 2.6. Let $\left(M_{i}, g_{i}\right)$ be a convergent sequence of Riemannian manifolds in $\mathcal{M}(n, D)$ (with respect to the measured Gromov-Hausdorff topology) such that $g_{i}$ satisfies the Assumption 1. Let $(M, g, \Phi)$ be the limit manifold. Suppose $(N, h)$ is a compact Riemannian manifold. Let $f_{i}:\left(M_{i}, g_{i}\right) \rightarrow(N, h)$ be a sequence of smooth harmonic maps such that $\left\|e_{g_{i}}\left(f_{i}\right)\right\|_{L^{\infty}}<C$, where $C$ is a constant independent of $i$. Then $f_{i}$ has a subsequence which converges to a map $f:\left(M, g, \Phi \mathrm{dvol}_{M}\right) \rightarrow(N, h)$ and this map is a smooth harmonic map.

Before we prove the Proposition 2.6, we first recall a few remarks from [Fuk88, Fuk89]. Then we prove Lemma 2.7 which is the main element in the proof of Proposition 2.6.

Remark 4. In [Fuk89] Fukaya proves that with the extra regularity assumption (9) on $g_{i}$, $\left(M_{i}, g_{i}, \frac{\text { dvol }_{M_{i}}}{\operatorname{vol}\left(M_{i}\right)}\right)$ converges to a smooth Riemannian manifold, with the smooth pair $(g, \Phi)$. See Lemma 2.1 in [Fuk89]. By Theorem 1.15, we know that for $i$ large enough, there is a fibration map $\psi_{i}: M_{i} \rightarrow M$. Since $g_{i}$ is an invariant metric, there exist metrics $g_{i}^{M}$ on $M$ such that the maps $\psi_{i}:\left(M_{i}, g_{i}\right) \rightarrow\left(M, g_{i}^{M}\right)$ are Riemannian submersions and $g_{i}^{M}$ converges to $g$ as in Theorem 1.13 .

Remark 5 (Fukaya [Fuk88, Fuk89]). Take an arbitrary point $p_{0}$ in $M$ and choose $p_{i} \in$ $\psi_{i}^{-1}\left(p_{0}\right)$. By $\left|\sec _{g_{i}}\right| \leq 1$, at point $p_{i}$ on $M_{i}$ the conjugate radius 11 is greater than some constant name it $\rho$. We name the pullback of the Riemannian metric $g_{i}$ by the exponential map, $\exp _{p_{i}}$ at $p_{i}, \tilde{g}_{i}$. Therefore the injectivity radius at 0 is at least the conjugate radius at $p_{i}$ (see Corollary 2.2.3 in [Ron10]).

Consider the ball $B=B(0, \rho)$ in $T_{p_{i}} M_{i}$ with the metric $\tilde{g}_{i}$. By virtue of the regularity assumption on $g_{i}, \tilde{g}_{i}$ will converge to some $g_{0}$ in the $C^{\infty}$-topology. There are local groups $G_{i}$ converging to a Lie group germ $G$ such that

1. $G_{i}$ act by isometries on the pointed metric spaces $\left(\left(B, \tilde{g}_{i}\right), 0\right)$.

2. $\left(\left(B, \tilde{g}_{i}\right), 0\right) / G_{i}$ is isometric to a neighborhood of $p_{i}$ in $M_{i}$.

3. $G$ acts by isometries on the pointed metric space $\left(\left(B, g_{0}\right), 0\right)$.

4. $\left(\left(B, g_{0}\right), 0\right) / G$ is isometric to a neighborhood of $p_{0}$ in $M$ and the action of $\mathrm{G}$ is free.

It follows that there is a neighborhood $U$ of $p_{0}$ in $M$ and a $C^{\infty}$ map $s: U \rightarrow B$ such that

i. $s\left(p_{0}\right)=0$.

\footnotetext{
${ }^{1}$ The conjugate domain at a point $p$ in a Riemannian manifold $M$ is the largest star shaped domain in which $d \exp _{p}$ is non-singular and the conjugate radius is the radius of the largest ball in the conjugate domain at $p$.
} 
ii. $P \circ s=I d$, where $P$ denotes the composition of the projection map and the above mentioned isometry in 4.

iii. $d_{\left(B, g_{0}\right)}(s(q), 0)=d_{M}\left(q, p_{0}\right)$ holds for $q \in M$.

Therefore there is some constant, which we again name $\rho$, independent of $i$ such that, $M=$ $\bigcup_{j=1}^{m} B \frac{\rho}{2}\left(x_{j}, M\right)$ and $B \frac{\rho}{2}\left(x_{j}, M\right)$ satisfies the preceding conditions and we can construct a smooth section $s_{i, j}: B_{\frac{\rho}{2}}\left(x_{j}, M\right) \rightarrow M_{i}$ of $\psi_{i}$, such that

$$
\frac{\left|\left(s_{i, j}\right)_{*}(v)\right|}{|v|}<C
$$

for each $v \in T B_{\frac{\rho}{2}}\left(x_{j}, M\right)$. Here $C$ is a constant independent of $i$. Hereafter we let $p_{i, j}=\psi_{i}^{-1}\left(x_{j}\right)$ and by $B\left(p_{i, j}\right)$ we mean a ball centered at $p_{i, j}$ with radius $\rho$ in $T_{p_{i, j}} M_{i}$. See section 3 in [Fuk88] and section 2 in [Fuk89.

Now we show that $f_{i}$ s are almost constant on the fibers of $M_{i}$. The following lemma is similar to Lemma 4.3 in [Fuk87]. In the following lemma $\left(M_{i}, g_{i}\right)$ is a convergent sequence in $\mathcal{M}(n, D)$ such that $g_{i}$ satisfies only (9) and $N$ is a compact Riemannian manifold.

Lemma 2.7. Let $h_{i}: M_{i} \rightarrow I(N) \subset \mathbb{R}^{q}$ be smooth maps which satisfy the Euler-Lagrange equation (11). Suppose $v_{i} \in T_{p}\left(M_{i}\right)$ satisfies $\left(\psi_{i}\right)_{*}\left(v_{i}\right)=0$, where $\psi_{i}$ is the fibration map and $v_{i}^{\prime}, v_{i}^{\prime \prime} \in T_{p}\left(M_{i}\right)\left(p \in B_{2 \rho / 3}\left(p_{i, j}, M_{i}\right)\right)$. Then we have

$$
\begin{aligned}
\left|v_{i} \cdot h_{i}\right| & \leq C_{1} \cdot \epsilon_{i}^{\prime} \cdot\left|v_{i}\right| \cdot\left(\left\|\Delta h_{i}\right\|_{L^{\infty}}+\left\|h_{i}\right\|_{L^{\infty}}\right), \\
\left|v_{i}^{\prime} \cdot v_{i}^{\prime \prime} \cdot h_{i}\right| & \leq C_{2} \cdot\left|v_{i}^{\prime}\right| \cdot\left|v_{i}^{\prime \prime}\right| \cdot\left(\left\|\Delta h_{i}\right\|_{L^{\infty}}+\left\|h_{i}\right\|_{L^{\infty}}\right),
\end{aligned}
$$

where $C_{1}$ and $C_{2}$ are some constants independent of $i$ and $\epsilon_{i}^{\prime}$ is a sequence converging to zero. Also $v_{i} \cdot h_{i}=d h_{i}\left(v_{i}\right)$ denotes the derivative of $h_{i}$ in the direction of $v_{i}$.

Proof. We put $\Phi_{i, j}=\exp _{p_{i, j}}: B\left(p_{i, j}\right) \rightarrow M_{i}, \tilde{g}_{i, j}=\Phi_{i, j_{*}}\left(g_{i}\right)$ and $a=\Phi_{i, j}^{-1}(p)$. We also denote $h_{i} \circ \Phi_{i, j}$ by $h_{i, j}$.

From the Schauder estimates for elliptic equations (see Theorem 1.7) we have

$$
\left\|h_{i, j}\right\|_{C^{1, \alpha}} \leq C^{\prime} \cdot\left(\left\|\Delta h_{i, j}\right\|_{L^{\infty}}+\left\|h_{i, j}\right\|_{L^{\infty}}\right),
$$

and hence

$$
\left\|v_{i}^{\prime} \cdot h_{i, j}\right\|_{C^{\alpha}} \leq C^{\prime} \cdot\left(\left\|\Delta h_{i}\right\|_{L^{\infty}}+\left\|h_{i}\right\|_{L^{\infty}}\right),
$$

where $C^{\prime}$ depends on the metric $\tilde{g}_{i, j}$. Since $\Phi_{i, j}$ is an isometry, by the composition formula (see formula 1.4.1 in [Xin96]), we have $\Delta h_{i, j}(x)=\Delta h_{i}\left(\Phi_{i, j}(x)\right.$ ). Also from (13), and the fact that $\tilde{g}_{i, j}$ converges in $C^{\infty}$

$$
\left\|\Pi\left(h_{i, j}\right)\left(d h_{i, j}, d h_{i, j}\right)\right\|_{C^{\alpha}} \leq C^{\prime \prime} \cdot\left(\left\|\Delta h_{i}\right\|_{L^{\infty}}+\left\|h_{i}\right\|_{L^{\infty}}\right),
$$

where $C^{\prime \prime}$ is a constant independent of $i$. By equation (11), we have

$$
\left\|\Delta h_{i, j}\right\|_{C^{\alpha}} \leq C^{\prime \prime} \cdot\left(\left\|\Delta h_{i}\right\|_{L^{\infty}}+\left\|h_{i}\right\|_{L^{\infty}}\right) .
$$


Using Schauder estimates for second derivative, we have

$$
\left\|h_{i, j}\right\|_{C^{2, \alpha}} \leq C \cdot\left(\left\|\Delta h_{i}\right\|_{L^{\infty}}+\left\|h_{i}\right\|_{L^{\infty}}\right),
$$

for some $C$ independent of $i$ and (12) follows.

Now we prove (11) by contradiction. Assume $\left|v_{i}\right|=1$. Let $\sigma^{i}(t)=\exp _{p}^{F_{i}}\left(t v_{i}\right)$ be a geodesic in the fiber containing $p, F_{i} \subset M_{i}$ such that $\left.\frac{d}{d t}\right|_{t=0} \sigma^{i}(t)=v_{i}$. For $0 \leq t \leq \frac{\rho}{5}$ this curve has a lift $l^{i}(t) \subset B\left(p_{i, j}\right)$ such that $\Phi_{i, j}\left(l^{i}(t)\right)=\sigma^{i}(t)$. We have

$$
d\left(\sigma_{i}(t), p\right) \leq \operatorname{diam}\left(F_{i}\right) \leq \epsilon_{i} .
$$

By contradiction we assume that there is subsequence of $h_{i}$ and a positive number $A$ such that

$$
\left|v_{i} \cdot h_{i, j}\right|>A \cdot\left(\left\|\Delta h_{i}\right\|_{L^{\infty}}+\left\|h_{i}\right\|_{L^{\infty}}\right)
$$

We know that

$$
v_{i} \cdot h_{i}=v_{i} \cdot h_{i, j}=\left.\frac{d}{d t}\right|_{t=0} h_{i, j} \circ l^{i}(t) .
$$

There exist $\beta>0$ and $\delta>0$ independent of $i$ such that for any $t<\delta$, we have

$$
\left|h_{i, j} \circ l^{i}(t)-h_{i, j}(a)\right|>\beta \cdot t \cdot\left(\left\|\Delta h_{i}\right\|_{L^{\infty}}+\left\|h_{i}\right\|_{L^{\infty}}\right) .
$$

To explain this, let $h_{i, j} \circ l^{i}(t)=q_{i, j}(t)$. We know from (15) that

$$
\left|\frac{d}{d t}\right|_{t=0} q_{i, j}^{\prime}(t) \mid \leq C\left(\left\|\Delta h_{i}\right\|_{L^{\infty}}+\left\|h_{i}\right\|_{L^{\infty}}\right)
$$

so for some fixed $\delta$ and $0<t<\delta$ we have

$$
\left|q_{i, j}^{\prime}(t)-q_{i, j}^{\prime}(0)\right| \leq C^{\prime} \cdot t \cdot\left(\left\|\Delta h_{i}\right\|_{L^{\infty}}+\left\|h_{i}\right\|_{L^{\infty}}\right) .
$$

On the other hand we have

$$
\left|q_{i, j}^{\prime}(0)\right|>A \cdot\left(\left\|\Delta h_{i}\right\|_{L^{\infty}}+\left\|h_{i}\right\|_{L^{\infty}}\right),
$$

so for $\delta$ small enough and $t<\delta$ we have

$$
\left|q_{i, j}^{\prime}(t)\right|>\beta \cdot\left(\left\|\Delta h_{i}\right\|_{L^{\infty}}+\left\|h_{i}\right\|_{L^{\infty}}\right) .
$$

Therefore

$$
\left|q_{i, j}(t)-q_{i, j}(0)\right|=\left|q_{i, j}^{\prime}\left(\theta_{i}\right) \cdot t\right|>\beta \cdot t \cdot\left(\left\|\Delta h_{i}\right\|_{L^{\infty}}+\left\|h_{i}\right\|_{L^{\infty}}\right),
$$

from which (16) follows.

There exists $b \in B\left(p_{i, j}\right)$, such that $d(a, b)<\epsilon_{i}$ and $\Phi_{i, j}\left(l_{i}\left(\delta^{\prime}\right)\right)=b$. For a fixed $\delta^{\prime}<\delta$ we have

$$
\left|h_{i, j}(b)-h_{i, j}(a)\right|>\beta \cdot \delta^{\prime} \cdot\left(\left\|\Delta h_{i}\right\|_{L^{\infty}}+\left\|h_{i}\right\|_{L^{\infty}}\right) .
$$


If we fix $\left\{\xi_{k}\right\}_{k=0}^{k=n}$ as a coordinate system at the point $a \in B\left(p_{i, j}\right)$, for some $b^{\prime} \in B\left(p_{i, j}\right)$ we have

$$
\sum_{k=0}^{k=n} \frac{\partial h_{i, j}}{\partial \xi^{k}}>C \cdot \beta \cdot \frac{\delta^{\prime}}{\epsilon_{i}} \cdot\left(\left\|\Delta h_{i}\right\|_{L^{\infty}}+\left\|h_{i}\right\|_{L^{\infty}}\right),
$$

and this contradicts (14).

Now we prove Proposition 2.6.

Proof of Proposition [2.6. As we assumed $\left\|e\left(f_{i}\right)\right\|_{L^{\infty}}<c$ and by the Euler-Lagrange equation and Corollary 1.6, we have that $\left\|\Delta I \circ f_{i}\right\|_{L^{\infty}}$ is uniformly bounded. Moreover, $\left\|I \circ f_{i}\right\|_{L^{\infty}}$ is uniformly bounded. Using (11), the maps $f_{i}$ s are equicontinuous. By Lemma 1.12, there is a limit map $f: M \rightarrow N$ which is continuous.

We consider the following maps on $M$,

$$
\tilde{f}_{i}=\sum \beta_{j} \cdot\left(I \circ f_{i}\right) \circ s_{i, j},
$$

where $\beta_{j}$ is an arbitrary $C^{\infty}$ partition of unity associated to $B_{\frac{\rho}{2}}\left(x_{j}, M\right), s_{i, j}$ is the section associated to $\psi_{i}$ as mentioned in Remark 5. Along a subsequence, which we again denote by $f_{i}$, we have

$$
\lim _{i \rightarrow \infty} f_{i}\left(s_{i, j}(x)\right)=f(x) \quad \text { for } x \in B_{\frac{\rho}{2}}\left(x_{j}, M\right)
$$

and also

$$
\lim _{i \rightarrow \infty} \tilde{f}_{i}(x)=I \circ f(x) \quad \text { for } x \in B_{\frac{\rho}{2}}\left(x_{j}, M\right) .
$$

Since the energy density of $f_{i}$ is bounded and also $s_{i, j}$ satisfies (10), we have $\left\|e\left(\tilde{f}_{i}\right)\right\|_{L^{\infty}}$ is uniformly bounded. By the same argument as above, $\left\|\tilde{f}_{i}\right\|_{C^{1}}$ is bounded and $\tilde{f}_{i}$ converge uniformly to $I \circ f$. Moreover $\psi_{i}$ has bounded second fundamental form (see Theorem 2.6 in [CFG92]) and the same is true for $s_{i, j}$. So $\tilde{f}_{i}$ has bounded $C^{2}$-norm and there is a subsequence of $\tilde{f}_{i}$ which converges to $I \circ f$ in the $C^{1}$-topology.

Choose a local orthonormal frame $\left\{\bar{e}_{k}\right\}_{k=1}^{m}$ on $\left(M, g_{i}^{M}\right)$. Denote its horizontal lift on $\left(M_{i}, g_{i}\right)$ by $\left\{e_{k}\right\}_{k=1}^{m}$. Suppose $\left\{e_{t}\right\}_{t=m+1}^{n}$ is a local orthonormal frame field of the fiber $F_{i}$ in $M_{i}$ such that $\left\{e_{k}, e_{t}\right\}$ form a local orthonormal frame field in $M_{i}$ (note that we omit the index $i$ for the orthonormal frame fields on $\left(M_{i}, g_{i}\right)$ and $\left(M, g_{i}^{M}\right)$ ). Our aim is to show that $f$ is also weakly harmonic.

Lemma 2.8. We have

$$
\lim _{i \rightarrow \infty}\left|\left\langle d I \circ f_{i}, d \eta_{i}\right\rangle(p)-\left\langle d \tilde{f}_{i}, d \eta\right\rangle\left(\psi_{i}(p)\right)\right|=0,
$$

where $\eta: M \rightarrow \mathbb{R}^{q}$, is a $C^{\infty}-$ map $\eta_{i}=\eta \circ \psi_{i}$, and $p$ in $M_{i}$.

Proof. By inequality (11),

$$
\left|\left\langle d I \circ f_{i}, d \eta_{i}\right\rangle(p)-\sum_{k=1}^{m}\left\langle d i \circ f_{i}\left(e_{k}\right), d \eta_{i}\left(e_{k}\right)\right\rangle(p)\right| \leq C_{1} \cdot \epsilon_{i}^{\prime}
$$


for $i$ large enough where $C_{1}$ is a constant independent of $i$. Let $F_{i}$ denote the fiber containing $p$ and choose a point $q$ in $F_{i}$. By (12), and since $\operatorname{diam}\left(F_{i}\right) \leq \epsilon_{i}$

$$
\left|d I \circ f_{i}\left(e_{k}\right)(p)-d I \circ f_{i}\left(e_{k}\right)(q)\right| \leq C_{2} \cdot \epsilon_{i},
$$

and so

$$
\left|d I \circ f_{i}\left(e_{k}\right)(p)-d I \circ f_{i}\left(e_{k}\right)\left(s_{i, j} \circ \psi_{i}(p)\right)\right| \leq C_{2} \cdot \epsilon_{i} .
$$

Because $\psi_{i} \circ s_{i, j}=\mathrm{Id}$, for $x \in M$ we have

$$
\psi_{i *}\left(e_{k}\left(s_{i, j}(x)\right)-s_{i, j_{*}}\left(\bar{e}_{k}(x)\right)\right)=0 .
$$

By inequality (10), we have

$$
\left|e_{k}\left(s_{i, j}(x)\right)-s_{i, j_{*}}\left(\bar{e}_{k}(x)\right)\right| \leq C_{3},
$$

for some constant $C_{3}$ and therefore by (11),

$$
\left|d I \circ f_{i}\left(e_{k}\right)(p)-d\left(I \circ f_{i}\right) \circ s_{i, j_{*}}\left(\bar{e}_{k}\right)\left(\psi_{i}(p)\right)\right| \leq C_{4} \cdot \epsilon_{i} .
$$

From the convergence of $f_{i} \circ s_{i, j}$ to $f$, we have

$$
\lim _{i \rightarrow \infty}\left|\sum d \beta_{j} \cdot\left(I \circ f_{i}\right) \circ s_{i, j}-\sum d \beta_{j} \cdot(I \circ f)\right|=0,
$$

So

$$
\lim _{i \rightarrow \infty}\left|d \tilde{f}_{i}-\sum \beta_{j} \cdot d\left(\left(I \circ f_{i}\right) \circ s_{i, j}\right)\right|=0 .
$$

Since $\sum_{j} \beta_{j}=1$ we finally have

$$
\lim _{i \rightarrow}\left|\left\langle d I \circ f_{i}, d \eta_{i}\right\rangle(p)-\left\langle d \tilde{f}_{i}, d \eta\right\rangle\left(\psi_{i}(p)\right)\right|=0 .
$$

Lemma 2.9. We have

$$
\lim _{1 \rightarrow \infty}\left|\Pi\left(f_{i}\right)(p)\left(d I \circ f_{i}, d I \circ f_{i}\right)-\Pi\left(\tilde{f}_{i}\right)\left(\psi_{i}(p)\right)\left(d \tilde{f}_{i}, d \tilde{f}_{i}\right)\right|=0 .
$$

Proof. By the proof of the above lemma, we have

$$
\lim _{i \rightarrow \infty}\left|d f_{i}(p)-d \tilde{f}_{i}\left(\psi_{i}(p)\right)\right|=0 .
$$

By the same argument as in Lemma 2.8 we can conclude

$$
\begin{gathered}
\left|\Pi\left(f_{i}\right)(p)\left(d I \circ f_{i}, d I \circ f_{i}\right)-\Pi\left(\tilde{f}_{i}\right)\left(\psi_{i}(p)\right)\left(d \tilde{f}_{i}, d \tilde{f}_{i}\right)\right| \\
\leq C \cdot\left|d f_{i}(p)-d \tilde{f}_{i}\left(\psi_{i}(p)\right)\right| .
\end{gathered}
$$


The map $\tilde{f}_{i}:\left(M, g_{i}^{M}, \operatorname{dvol}_{g_{i}^{M}}\right) \rightarrow \mathbb{R}^{q}$ converges in $C^{1}$ to the map $I \circ f$, and $\Phi_{i}$ converges to $\Phi$ in the $C^{\infty}$ topology. Also $\left(M, g_{i}^{M}\right)$ converges to $(M, g)$ in $\mathcal{M}(n, D, v)$. Therefore we have

$$
\left|\int_{M} \Xi_{g_{i}^{M}}\left(\eta, \tilde{f}_{i}\right) \Phi_{i} \operatorname{dvol}_{g_{i}^{M}}-\int_{M} \Xi(\eta, f) \Phi \operatorname{dvol}_{g}\right| \leq C \cdot \epsilon_{i},
$$

where $\Xi(\cdot, \cdot)$ is defined by (3). By Lemma 2.8 and 2.9, we have

$$
\lim _{i \rightarrow \infty}\left|\int_{M_{i}} \Xi_{g_{i}}\left(\eta_{i}, f_{i}\right) \frac{\mathrm{dvol}_{M_{i}}}{\operatorname{vol}\left(M_{i}\right)}-\int_{M} \Xi_{g_{i}^{M}}\left(\eta, \tilde{f}_{i}\right) \psi_{i *}\left(\frac{\mathrm{dvol}_{M_{i}}}{\operatorname{vol}\left(M_{i}\right)}\right)\right|=0 .
$$

It follows that

$$
\lim _{i \rightarrow \infty} \int_{M_{i}} \Xi_{g_{i}}\left(\eta_{i}, f_{i}\right) \frac{\mathrm{dvol}_{M_{i}}}{\operatorname{vol}\left(M_{i}\right)}=\int_{M} \Xi_{g}(\eta, f) \Phi \mathrm{dvol}_{M}
$$

Therefore $f$ is weakly harmonic and since it is continuous, it is also a smooth harmonic map.

Now we prove Case II without considering Assumption [1.

Proof of Proposition 2.5. By Remark 2 we can obtain a $C^{1}$-close metric $g_{i}(\epsilon)$ to $g_{i}$ which satisfies (9) and such that the map $\psi_{i}:\left(M_{i}, g_{i}(\epsilon)\right) \rightarrow\left(M, \psi_{i_{*}}\left(g_{i}(\epsilon)\right)\right)$ is a Riemannian submersion.

For small $\epsilon$, let $M(\epsilon)$ be the Gromov-Hausdorff limit of a subsequence of $\left(M_{i}, g_{i}(\epsilon)\right)$. By Lemma 2.3 in [Fuk88, $\left(M_{i}, g_{i}(\epsilon)\right)$ and $(M(\epsilon), g(\epsilon))$ converge to $\left(M_{i}, g_{i}\right)$ and $(M, g)$ in $\mathcal{M}(n, D, v)$ respectively.

The map $f_{i}:\left(M_{i}, g_{i}\right) \rightarrow(N, h)$ is harmonic and since $g_{i}(\epsilon)$ is $C^{1}$-close to $g$, we have

$$
\left|\Xi_{g_{i}}\left(f_{i}, \eta_{i}\right)-\Xi_{g_{i}(\epsilon)}\left(f_{i}, \eta_{i}\right)\right| \leq C \cdot \epsilon .
$$

By (18), we have

$$
\lim _{i \rightarrow \infty}\left|\int_{M_{i}} \Xi_{g_{i}(\epsilon)}\left(f_{i}, \eta_{i}\right) \frac{\operatorname{dvol}_{\left(M_{i}, g_{i}(\epsilon)\right)}}{\operatorname{vol}\left(\left(M_{i}, g_{i}(\epsilon)\right)\right.}-\int_{M(\epsilon)} \Xi_{g(\epsilon)}(f, \eta) \cdot \Phi(\epsilon) \operatorname{dvol}_{M(\epsilon)}\right|=0,
$$

and finally since $g(\epsilon)$ converges to $g$ in the $C^{1, \alpha}$-topology, we have the desired result.

2.3. Case III: Collapsing to a singular space. Now we are going to investigate the general case when the sequence converges to a singular space. This means that $\left(M_{i}, g_{i}\right)$ in $\mathcal{M}(n, D)$ converges to some metric space $(X, d)$. First we recall the following remark from Fuk87.

Remark 6 (Fukaya Fuk87, $\S 7$ ). Let $Y$ be a Riemannian manifold on which $O(n)$ acts by isometry, and let $\theta: Y \rightarrow[0, \infty)$ be an $O(n)$-invariant smooth function. Put $X=Y / O(n)$. Let $p: Y \rightarrow X$ be the natural projection, $\bar{\theta}: X \rightarrow[0, \infty)$ the function induced from $\theta$, and $S(X)$ the set of all singular points of $X$. The set $S(X) \subset X$ has a well defined normal 
bundle on the codimension 2 strata $(X=Y / O(n)$ is a Riemannian polyhedron and $S(X)$ is a subset of the $(n-2)$-skeleton of $X)$. Set

$$
\operatorname{Lip}(X, S(X))=\{u \in \operatorname{Lip}(X) \mid v \cdot u=0 \text { if } v \text { is perpendicular to } S(X)\} .
$$

Define $Q_{1}: \operatorname{Lip}(Y) \times \operatorname{Lip}(Y) \rightarrow[0, \infty)$ and $Q_{2}: \operatorname{Lip}(X, S(X)) \times \operatorname{Lip}(X, S(X)) \rightarrow[0,1)$ by

$$
\begin{aligned}
Q_{1}(\tilde{k}, \tilde{h}) & =\int_{Y} \theta \cdot\langle\nabla \tilde{k}, \nabla \tilde{h}\rangle \mathrm{dvol}_{Y}, \\
Q_{2}(k, h) & =\int_{X} \bar{\theta} \cdot\langle\nabla k, \nabla h\rangle d \mu_{g} .
\end{aligned}
$$

It is easy to see that $f \circ p \in \operatorname{Lip}(Y)$ for each $f$ contained in $\operatorname{Lip}(X, S(X))$. Define $p^{*}$ : $\operatorname{Lip}(X, S(X)) \rightarrow \operatorname{Lip}(Y)$ by $p^{*}(f)=f \circ p$. Let $\operatorname{Lip}_{O(n)}(Y)$ be the set of all $O(n)$-invariant elements of $\operatorname{Lip}(Y)$. Then, we can easily prove the following

Lemma 2.10. $p^{*}$ is a bijection between $\operatorname{Lip}(X, S(X))$ and $\operatorname{Lip}_{O(n)}(Y)$. For elements $f$ and $k$ of $\operatorname{Lip}(X, S(X))$, we have

$$
Q_{1}(f, k)=Q_{2}\left(p^{*}(f), p^{*}(k)\right)
$$

and

$$
\int_{Y} \theta \cdot p^{*}(f) p^{*}(k) \operatorname{dvol}_{Y}=\int_{X} \bar{\theta} \cdot f k d \mu_{g}
$$

Now we prove the main theorem of this paper.

Proof of Theorem 0.1 . We denote by $\left(Y, g, \Phi_{Y} \mathrm{dvol}_{Y}\right)$ the limit space of the frame bundles over $M_{i}$, and by $(X, d, \nu)$ the limit space of $M_{i}$ with respect to the measured GromovHausdorff topology. We know $(X, \nu)=\left(Y, \Phi_{Y}{\mathrm{~d} v o l_{Y}}\right) / O(n)$ (see Subection [1.6 ). The projection $p_{i}:\left(F\left(M_{i}\right), \tilde{g}_{i}\right) \rightarrow\left(M_{i}, g_{i}\right)$ is a Riemannian submersion with totally geodesic fibers. So using the reduction formula the map $\bar{f}_{i}=f_{i} \circ p_{i}$ is harmonic on $F\left(M_{i}\right)$ and it is invariant under the action of $O(n)$. Furthermore $\left\|e_{\tilde{g}_{i}}\left(\bar{f}_{i}\right)\right\|_{\infty}$ is bounded ( $p_{i}$ is a Riemannian submersion). Using Case II, $\bar{f}_{i}$ converge to some map $\bar{f}$ on $\left(Y, g, \Phi_{Y} \mathrm{dvol}_{Y}\right)$. The map $\bar{f}$ satisfies

$$
\int_{Y} \Xi_{g}(\bar{f}, \eta) \Phi_{Y} \mathrm{dvol}_{Y}=0
$$

where $\eta$ is a test function. The map $\bar{f}$ is also $O(n)$ invariant and continuous. Consider a quotient map $f$ such that $\bar{f}=p^{*}(f)$. First we show that $f$ is in $\mathcal{H}^{1}((X, \nu), N)$. By the argument in Case II, $\bar{f}$ is in $\mathcal{H}^{1}\left(\left(Y, \Phi_{Y} \operatorname{dvol}_{Y}\right), N\right)$ and so by equation (19), $f$ has finite energy. Now we show that $f$ is weakly harmonic on $(X, \nu)$. By equation (19), for $\eta$ in $\operatorname{Lip}(X, S(X))$

$$
\int_{Y}\left\langle\nabla I \circ \bar{f}, \nabla p^{*}(\eta)\right\rangle \Phi_{Y} \operatorname{dvol}_{Y}=\int_{X}\langle\nabla I \circ f, \nabla \eta\rangle \Phi_{X} d \mu_{g}
$$


Furthermore

$$
\begin{array}{r}
\int_{Y}\left\langle\Pi(\bar{f})\left(\nabla^{g}(I \circ \bar{f}), \nabla^{g}(I \circ \bar{f})\right), p^{*}(\eta)\right\rangle \Phi_{Y} \mathrm{dvol}_{Y} \\
=\int_{X}\langle\Pi(f)(\nabla(I \circ f), \nabla(I \circ f)), \eta\rangle \Phi_{X} d \mu_{g},
\end{array}
$$

and since $\Phi_{Y}=p^{*}\left(\Phi_{X}\right)$

$$
\int_{Y} \Xi_{g}\left(\bar{f}, p^{*}(\eta)\right) \Phi_{Y} \mathrm{dvol}_{Y}=\int_{X} \Xi(f, \eta) \Phi_{X} d \mu_{g}
$$

which shows that $f: X \rightarrow N$ is a weakly harmonic map.

\section{Appendix: Convergence of tension field.}

In this section we study convergence of the tension fields of the maps $f_{i}, \tau\left(f_{i}\right)$, under the assumptions of Proposition 2.6.

Assume $\left(M_{i}, g_{i}\right), f_{i}, N$ to be as in Proposition 2.6. Moreover consider the following assumption

Assumption 2. The section $s_{i, j}$ is almost harmonic,

$$
\left|\tau\left(s_{i, j}\right)\right| \leq C \cdot \epsilon_{i}^{\prime \prime}
$$

and also

$$
\left|\nabla_{\bar{X}} d s_{i, j}(X)\right| \leq C \cdot \epsilon_{i}^{\prime \prime},
$$

where $X$ is a smooth vector field on $M$ and $\bar{X}$ is its horizontal lift and $\epsilon_{i}^{\prime \prime}$ is a sequence which converges to zero.

Using Assumption 1 and by Theorem 1.4 we have

$$
\begin{aligned}
\tau\left(f_{i}\right) & =\left(\nabla_{e_{k}} d f_{i}\right) e_{k}+\left(\nabla_{e_{t}} d f_{i}\right) e_{t} \\
& =\left(\nabla_{e_{k}} d f_{i}\right) e_{k}+\nabla_{f_{*}\left(e_{t}\right)} f_{i_{*}}\left(e_{t}\right) \\
& -f_{i_{*}}\left(\nabla_{e_{t}} e_{t}\right)^{H}-f_{i_{*}}\left(\nabla_{e_{t}} e_{t}\right)^{V} \\
& =\left(\nabla_{e_{k}} d f_{i}\right) e_{k}-f_{i_{*}}\left(\mathrm{H}_{i}\right)+\tau\left(f_{i}^{\perp}\right)
\end{aligned}
$$

where $\left\{e_{k}, e_{t}\right\}$ and $\bar{e}_{k}$ are as in the proof of Proposition 2.6. $f_{i}{ }^{\perp}$ denotes the restriction of $f_{i}$ to the fibers $F_{i}$, and $\mathrm{H}_{i}$ is the mean curvature vector of the submanifold $F_{i}$.

We investigate how each term of the equation above behaves as $f_{i}$ converges to $f$.

Lemma 3.1. We have

$$
\lim _{i \rightarrow \infty}\left|d I\left(\nabla_{e_{k}} d f_{i}\right) e_{k}(p)-\left(\Delta_{g_{i}^{M}} \tilde{f}_{i}-\Pi\left(\tilde{f}_{i}\right)\left(d \tilde{f}_{i}, d \tilde{f}_{i}\right)\right)\left(\psi_{i}(p)\right)\right|=0
$$


Proof. By the discussion in the proof of Proposition 2.6, we know that $\tilde{f}_{i}$ converges to $f$ in the $C^{1}$-topology. Using the composition formula we have

$$
d I\left(B f_{i}\left(X_{1}, X_{2}\right)\right)=B\left(I \circ f_{i}\right)\left(X_{1}, X_{2}\right)-B\left(\pi_{N}\right)\left(d\left(I \circ f_{i}\right)\left(X_{1}\right), d\left(I \circ f_{i}\right)\left(X_{2}\right)\right),
$$

and so for $k=1, \ldots, n$,

$$
d I\left(\left(\nabla_{e_{k}} d f_{i}\right) e_{k}\right)=\left(\nabla_{e_{k}} d\left(I \circ f_{i}\right)\right) e_{k}-B\left(\pi_{N}\right)\left(d\left(I \circ f_{i}\right)\left(e_{k}\right), d\left(I \circ f_{i}\right)\left(e_{k}\right)\right) .
$$

First we show that

$$
\lim _{i \rightarrow \infty}\left|\nabla_{e_{k}} d\left(I \circ f_{i}\right) e_{k}(p)-\Delta_{g_{i}^{M}} \tilde{f}_{i}\left(\psi_{i}(p)\right)\right|=0
$$

By definition of $\tilde{f}_{i}$,

$$
\begin{aligned}
\left(\nabla_{\bar{e}_{k}} d \tilde{f}_{i}\right) \bar{e}_{k} & =\sum\left(d \beta_{j}\left(\bar{e}_{k}\right) \cdot d f_{i}\left(s_{i, j_{*}}\left(\bar{e}_{k}\right)\right)\right. \\
& \left.+\beta_{j} \cdot\left(\nabla_{\bar{e}_{k}} d\left(f_{i} \circ s_{i, j}\right)\right) \bar{e}_{k}+\triangle \beta_{j} \cdot f_{i} \circ s_{i, j}\right) .
\end{aligned}
$$

and again by the composition formula

$$
\tau\left(f_{i} \circ s_{i, j}\right)=B_{s_{i, j_{*}}\left(\bar{e}_{k}\right), s_{i, j_{*}}\left(\bar{e}_{k}\right)} f_{i}+d f_{i}\left(\tau\left(s_{i, j}\right)\right) .
$$

Since $f_{i} \circ s_{i, j}$ converges in $C^{1}$ to $f$

$$
\begin{gathered}
\lim _{i \rightarrow \infty}\left|\sum d \beta_{j}\left(\bar{e}_{k}\right) \cdot d f_{i}\left(s_{i, j_{*}}\left(\bar{e}_{k}\right)\right)\right|=0, \\
\lim _{i \rightarrow \infty} \sum \Delta \beta_{j} \cdot f_{i} \circ s_{i, j}(x)=\sum \triangle \beta_{j} \cdot f(x)=0 .
\end{gathered}
$$

Also, $\psi_{i_{*}}\left(e_{k}-s_{i, j_{*}}\left(\bar{e}_{k}\right)\right)=0$ and so $e_{k}-s_{i, j_{*}}\left(\bar{e}_{k}\right)$ is vertical. On the other hand

$$
\left|e_{k}-s_{i, j_{*}}\left(\bar{e}_{k}\right)\right| \leq \epsilon_{i}
$$

By inequality (11) and almost harmonicity of $s_{i, j}$ (21), the second term on the right hand side of (25) converges to zero. Again by inequality (12) and (22), we have

$$
\begin{gathered}
\lim _{i \rightarrow \infty}\left|\left(\nabla_{e_{k}} d f_{i}\right)\left(e_{k}-s_{i, j_{*}}\left(\bar{e}_{k}\right)\right)\right|=0 \\
\lim _{i \rightarrow \infty}\left|\left(\nabla_{\left(e_{k}-s_{i, j_{*}}\left(\bar{e}_{k}\right)\right)} d f_{i}\right) e_{k}\right|=0
\end{gathered}
$$

Finally

$$
\lim _{i \rightarrow \infty}\left|\left(\nabla_{e_{k}} d\left(I \circ f_{i}\right)\right) e_{k}(p)-\left(\nabla_{\bar{e}_{k}} d \tilde{f}_{i}\right) \bar{e}_{k}(\psi(p))\right|=0 .
$$

We have the same for the second term

$$
\lim _{i \rightarrow \infty}\left|\Pi\left(f_{i}\right)(p)\left(d f_{i}, d f_{i}\right)-\Pi\left(\tilde{f}_{i}\right)\left(\psi_{i}(p)\right)\left(d \tilde{f}_{i}, d \tilde{f}_{i}\right)\right|=0 .
$$

By the above lemma and $\psi_{i_{*}}\left(\frac{\operatorname{dvol}_{M_{i}}}{\operatorname{vol}\left(M_{i}\right)}\right)=\Phi_{i} \operatorname{dvol}_{M}$, we have

$$
\lim _{i \rightarrow \infty}\left|\int_{M_{i}}\left\langle d I\left(\left(\nabla_{e_{k}} d f_{i}\right) e_{k}\right), \eta_{i}\right\rangle \frac{\operatorname{dvol}_{M_{i}}}{\operatorname{vol}\left(M_{i}\right)}-\int_{M}\left\langle\Delta^{g_{i}^{M}} \tilde{f}_{i}-\Pi\left(\tilde{f}_{i}\right)\left(d \tilde{f}_{i}, d \tilde{f}_{i}\right), \eta\right\rangle \Phi_{i} \operatorname{dvol}_{g_{i}^{M}}\right|=0
$$


and we conclude

$$
\begin{gathered}
\lim _{i \rightarrow \infty} \int_{M_{i}}\left\langle d I\left(\left(\nabla_{e_{k}} d f_{i}\right) e_{k}\right), \eta_{i}\right\rangle \frac{\mathrm{dvol}_{M_{i}}}{\operatorname{vol}\left(M_{i}\right)} \\
=\int_{M}[\langle d f, d \eta\rangle+\langle d f(\nabla \ln \Phi)-\Pi(f)(d f, d f), \eta\rangle] \Phi \operatorname{dvol}_{M} .
\end{gathered}
$$

Here $\eta$ is a test map on $M$ and $\eta_{i}=\eta \circ \psi_{i}$. Now we will consider the second and third terms in the decomposition of $\tau\left(f_{i}\right)$.

Lemma 3.2. With the same assumptions as above

i. $\lim _{i \rightarrow \infty} \int_{M_{i}}\left\langle d f_{i}\left(\mathrm{H}_{i}\right), \eta_{i}\right\rangle \frac{\operatorname{dvol}_{M_{i}}}{\operatorname{vol}\left(M_{i}\right)}=-\int_{M}\langle d f(\nabla \ln \Phi), \eta\rangle \Phi \operatorname{dvol}_{M}$.

ii. $\lim _{i \rightarrow \infty}\left\|\tau\left(f_{i}^{\perp}\right)\right\|=0$.

Here $\mathrm{H}_{i}$ denotes the mean curvature vector of the fibers $F_{i}^{x}=\psi_{i}^{-1}(x)$.

Before we prove Lemma 3.2, we prove the following lemma which we need for the proof of part i.

Lemma 3.3. We have

$$
\int_{M} \eta d \ln \Phi(X) \Phi \operatorname{dvol}_{M}=-\lim _{i \rightarrow \infty} \int_{M_{i}} \eta\left\langle X, \mathrm{H}_{i}\right\rangle \frac{\operatorname{dvol}_{M_{i}}}{\operatorname{vol}\left(M_{i}\right)}
$$

Proof. Suppose $X$ is a smooth vector field on $M$ and $X_{i}$ its horizontal lift on $M_{i}$. The flow $\theta_{t}^{i}$ of $X_{i}$ sends fibers to fibers diffeomorphically. By the first variation formula

$$
\left.\frac{d}{d t}\right|_{t=0} \theta_{t}^{i^{*}}\left(\operatorname{dvol}_{F_{i}^{x}}\right)=-\int_{F_{i}^{x}}\left\langle X_{i}, \mathrm{H}_{i}^{x}\right\rangle \operatorname{dvol}_{F_{i}^{x}}
$$

Also

$$
\Phi_{i}(x)=\frac{\operatorname{vol}\left(\psi_{i}^{-1}(x)\right)}{\operatorname{vol}\left(M_{i}\right)}
$$

and by (28),

$$
d \Phi_{i}(X)(x)=-\int_{F_{i}^{x}}\left\langle X_{i}, \mathrm{H}_{i}^{x}\right\rangle \frac{\mathrm{dvol}_{F_{i}^{x}}}{\operatorname{vol}\left(M_{i}\right)}
$$

For an arbitrary $\eta$ in $C^{\infty}(M)$, we prove

$$
\int_{M} \eta d \Phi_{i}(X) \operatorname{dvol}_{g_{i}^{M}}=-\int_{M_{i}} \eta_{i}\left\langle X_{i}, \mathrm{H}_{i}\right\rangle \frac{\mathrm{dvol}_{M_{i}}}{\operatorname{vol}\left(M_{i}\right)}
$$

If we consider $\left(U_{\gamma}, h_{\gamma}\right)$ as a local trivialization of the fibration $\psi_{i}$, then

$$
\int_{M} \chi_{U_{\gamma}} d \Phi_{i}(X) \mathrm{dvol}^{g_{i}^{M}}=-\int_{U_{\gamma}} \int_{F_{i}^{x}} \chi_{U_{\gamma}}\left\langle X_{i}, \mathrm{H}_{i}^{x}\right\rangle \frac{\mathrm{dvol}_{i}^{x}}{\operatorname{vol}\left(M_{i}\right)} \mathrm{dvol}_{g_{i}^{M}},
$$

and so

$$
\int_{M} \chi_{U_{\gamma}} d \Phi_{i}(X) \mathrm{dvol}_{M}^{g_{i}^{M}}=-\int_{\psi_{i}^{-1}\left(U_{\gamma}\right)}\left\langle X_{i}, \mathrm{H}_{i}\right\rangle \frac{\mathrm{dvol}_{M_{i}}}{\operatorname{vol}\left(M_{i}\right)},
$$


where $\chi_{U_{\gamma}}$ denotes the characteristic function on $U_{\gamma}$ and so we have (29). The functions $\Phi_{i}$ goes to $\Phi$ in $C^{\infty}$ and also $\mathrm{dvol}^{g_{i}^{M}}$ goes to $\mathrm{dvol}_{M}$ as $i$ goes to infinity. Letting $i$ go to $\infty$ on the both sides of (29) and by the definition of weak derivatives

$$
\int_{M} \eta d \ln \Phi(X) \Phi \operatorname{dvol}_{M}=-\lim _{i \rightarrow \infty} \int_{M_{i}} \eta\left\langle X, \mathrm{H}_{i}\right\rangle \frac{\operatorname{dvol}_{M_{i}}}{\operatorname{vol}\left(M_{i}\right)} .
$$

Proof of Lamma 3.2. Part i follows directly from Lemma 3.3.

To prove part ii consider

$$
\tau\left(f_{i}^{\perp}\right)=\nabla_{f_{i_{*}}\left(e_{t}\right)} f_{i_{*}}\left(e_{t}\right)-f_{i_{*}}\left(\nabla_{e_{t}} e_{t}\right)^{V} .
$$

From (11) and (12)

$$
\begin{aligned}
\left|\nabla_{f_{i_{*}}\left(e_{t}\right)} f_{i_{*}}\left(e_{t}\right)\right| & <C \cdot \epsilon_{i}^{\prime}, \\
\left\|f_{i_{*}}\left(\nabla_{e_{t}} e_{t}\right)^{V}\right\|_{L^{\infty}} & <C \cdot \epsilon_{i}^{\prime}\left|\left(\nabla_{e_{t}} e_{t}\right)^{V}\right|
\end{aligned}
$$

where $C$ is a constant independent of $i$. It follows that

$$
\lim _{i \rightarrow \infty}\left\|\tau\left(f_{i}^{\perp}\right)\right\|=0 .
$$

\section{REFERENCES}

[Bes08] Arthur L. Besse. Einstein manifolds. Classics in Mathematics. Springer-Verlag, Berlin, 2008. Reprint of the 1987 edition.

[BMOR84] Josef Bemelmans, Min-Oo, and Ernst A. Ruh. Smoothing Riemannian metrics. Math. Z., 188(1):69-74, 1984.

[CC97] Jeff Cheeger and Tobias H. Colding. On the structure of spaces with Ricci curvature bounded below. I. J. Differential Geom., 46(3):406-480, 1997.

[CC00a] Jeff Cheeger and Tobias H. Colding. On the structure of spaces with Ricci curvature bounded below. II. J. Differential Geom., 54(1):13-35, 2000.

[CC00b] Jeff Cheeger and Tobias H. Colding. On the structure of spaces with Ricci curvature bounded below. III. J. Differential Geom., 54(1):37-74, 2000.

[CFG92] Jeff Cheeger, Kenji Fukaya, and Mikhael Gromov. Nilpotent structures and invariant metrics on collapsed manifolds. J. Amer. Math. Soc., 5(2):327-372, 1992.

[Che70] Jeff Cheeger. Finiteness theorems for Riemannian manifolds. Amer. J. Math., 92:61-74, 1970.

[EF01] J. Eells and B. Fuglede. Harmonic maps between Riemannian polyhedra, volume 142 of Cambridge Tracts in Mathematics. Cambridge University Press, Cambridge, 2001. With a preface by M. Gromov.

[ES64] James Eells, Jr. and J. H. Sampson. Harmonic mappings of Riemannian manifolds. Amer. J. Math., 86:109-160, 1964.

[FLZ09] Fuquan Fang, Xiang-Dong Li, and Zhenlei Zhang. Two generalizations of Cheeger-Gromoll splitting theorem via Bakry-Emery Ricci curvature. Ann. Inst. Fourier (Grenoble), 59(2):563$573,2009$.

[Fuk87] Kenji Fukaya. Collapsing of Riemannian manifolds and eigenvalues of Laplace operator. Invent. Math., 87(3):517-547, 1987.

[Fuk88] Kenji Fukaya. A boundary of the set of the Riemannian manifolds with bounded curvatures and diameters. J. Differential Geom., 28(1):1-21, 1988. 
[Fuk89] Kenji Fukaya. Collapsing Riemannian manifolds to ones with lower dimension. II. J. Math. Soc. Japan, 41(2):333-356, 1989.

[GP91] Karsten Grove and Peter Petersen. Manifolds near the boundary of existence. J. Differential Geom., 33(2):379-394, 1991.

[Gro81] Mikhael Gromov. Structures métriques pour les variétés riemanniennes, volume 1 of Textes Mathématiques [Mathematical Texts]. CEDIC, Paris, 1981. Edited by J. Lafontaine and P. Pansu.

[GS92] Mikhail Gromov and Richard Schoen. Harmonic maps into singular spaces and $p$-adic superrigidity for lattices in groups of rank one. Inst. Hautes Études Sci. Publ. Math., (76):165-246, 1992.

[GT83] David Gilbarg and Neil S. Trudinger. Elliptic partial differential equations of second order, volume 224 of Grundlehren der Mathematischen Wissenschaften [Fundamental Principles of Mathematical Sciences]. Springer-Verlag, Berlin, second edition, 1983.

[GW88] R. E. Greene and H. Wu. Lipschitz convergence of Riemannian manifolds. Pacific J. Math., 131(1):119-141, 1988.

[KS93] Nicholas J. Korevaar and Richard M. Schoen. Sobolev spaces and harmonic maps for metric space targets. Comm. Anal. Geom., 1(3-4):561-659, 1993.

[Lic69] André Lichnerowicz. Applications harmoniques et variétés Kähleriennes. Rend. Sem. Mat. Fis. Milano, 39:186-195, 1969.

[Lin99] Fang-Hua Lin. Gradient estimates and blow-up analysis for stationary harmonic maps. Ann. of Math. (2), 149(3):785-829, 1999.

[Lot03] John Lott. Some geometric properties of the Bakry-Émery-Ricci tensor. Comment. Math. Helv., 78(4):865-883, 2003.

[Mor05] Frank Morgan. Manifolds with density. Notices Amer. Math. Soc., 52(8):853-858, 2005.

[Mos05] Roger Moser. Partial regularity for harmonic maps and related problems. World Scientific Publishing Co. Pte. Ltd., Hackensack, NJ, 2005.

[MW11] Ovidiu Munteanu and Jiaping Wang. Smooth metric measure spaces with non-negative curvature. Comm. Anal. Geom., 19(3):451-486, 2011.

[Pet84] Stefan Peters. Cheeger's finiteness theorem for diffeomorphism classes of Riemannian manifolds. J. Reine Angew. Math., 349:77-82, 1984.

[Qia97] Zhongmin Qian. Estimates for weighted volumes and applications. Quart. J. Math. Oxford Ser. (2), 48(190):235-242, 1997.

[Ron10] Xiaochun Rong. Convergence and collapsing theorems in Riemannian geometry. In Handbook of geometric analysis, No. 2, volume 13 of Adv. Lect. Math. (ALM), pages 193-299. Int. Press, Somerville, MA, 2010.

[Sch84] Richard M. Schoen. Analytic aspects of the harmonic map problem. In Seminar on nonlinear partial differential equations (Berkeley, Calif., 1983), volume 2 of Math. Sci. Res. Inst. Publ., pages 321-358. Springer, New York, 1984.

[SZ11] Yan-Hui Su and Hui-Chun Zhang. Rigidity of manifolds with Bakry-Émery ricci curvature bounded below. Geometriae Dedicata, pages 1-11, 2011.

[Tay00] Michael E. Taylor. Tools for PDE, volume 81 of Mathematical Surveys and Monographs. American Mathematical Society, Providence, RI, 2000. Pseudodifferential operators, paradifferential operators, and layer potentials.

[Wu10] Jia-Yong Wu. Upper bounds on the first eigenvalue for a diffusion operator via Bakry-Émery Ricci curvature. J. Math. Anal. Appl., 361(1):10-18, 2010.

[WW09] Guofang Wei and Will Wylie. Comparison geometry for the Bakry-Émery Ricci tensor. J. Differential Geom., 83(2):377-405, 2009.

[Xin96] Yuanlong Xin. Geometry of harmonic maps. Progress in Nonlinear Differential Equations and their Applications, 23. Birkhäuser Boston Inc., Boston, MA, 1996. 